Stabilization of weakly charged microparticles using highly charged nanoparticles

David J. Herman

Thesis submitted to the faculty of the Virginia Polytechnic Institute and State University in partial fulfillment of the requirements for the degree of

Master of Science

In

Chemical Engineering

John Y. Walz

William A. Ducker

Richey M. Davis

August 4, 2011

Blacksburg, VA

Keywords: Colloidal stability, Nanoparticle adsorption, Colloidal aggregation, Patchy adsorption 


\title{
Stabilization of weakly charged microparticles using highly charged nanoparticles
}

\author{
David Herman
}

\begin{abstract}
An experimental investigation was conducted to evaluate the possible use of highlycharged spherical nanoparticles to stabilize an aqueous dispersion of weakly-charged microspheres. At low $\mathrm{pH}$ values, the surface of silica is weakly charged, which leads to flocculation of colloidal suspensions of silica microspheres. Binary solutions of weakly charged silica microspheres and highly charged polystyrene latex nanoparticles result in adsorption of the nanoparticles onto the surface of the silica microspheres. This effectively "recharges" the silica spheres, with effective zeta potentials increased to the range that is unfavorable for flocculation of microspheres in a silica-only solution. However, this does not guarantee stability, and comparisons between positively charged amidine latex nanoparticles and negatively charged sulfate latex nanoparticles indicate that the degree of coverage plays an important role in the restabilization. The sulfate latex nanoparticles do not cover the surface sufficiently, and though they seemingly provide sufficient charge, the weakly charged patches of the exposed silica substrate can lead to flocculation. The amidine latex nanoparticles, on the other hand, cover the surface more completely, and effectively prevent flocculation of the silica microspheres. The mechanisms responsible for this different adsorption and stabilizing behavior are not entirely understood, as both the amidine and sulfate latex nanoparticles are of similar size and the magnitude of the zeta potentials of the different particle types are comparable.
\end{abstract}




\section{Acknowledgements}

I want to thank Dr. John Walz for having me in his research group. I have really enjoyed the experience and have learned quite a lot in the past couple of years. I want to thank my thesis committee, Dr. William Ducker and Dr. Richey Davis. I greatly appreciate their willingness to work with me (and allow me to use instruments in their labs) and to accommodate my defense even while traveling this summer. I also want to thank my lab-mates. Wenle, I could not have done this project without his expertise in SEM. Greg and Shunxi, it has been a pleasure working and discussing various aspects of our research, and for always being willing to help whenever I needed a hand with anything. Finally, I want to thank my parents. They have always been supportive of me and I could not have made it this far in my education and life without them. 


\section{Table of Contents}

$\begin{array}{ll}\text { Chapter 1. Introduction } & 1\end{array}$

$\begin{array}{lr}\text { Chapter 2. Materials and Experimental methods } & 8\end{array}$

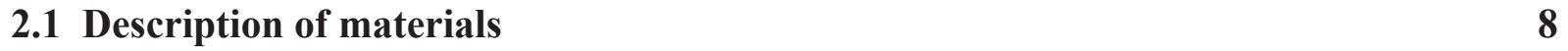

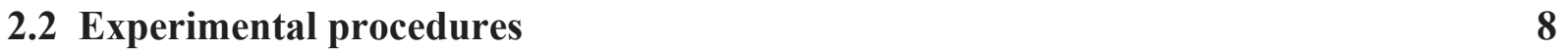

$\begin{array}{ll}\text { 2.2.1 Zeta potential measurements } & 8\end{array}$

$\begin{array}{ll}\text { 2.2.2 ZetaSpin measurements } & \mathbf{1 0}\end{array}$

2.2.3 Measurement of nanoparticle adsorption via scanning electron microscopy $\mathbf{1 1}$

2.2.4 Measurement of nanoparticle adsorption with quartz crystal microbalance $\mathbf{1 3}$

2.2.5 Microsphere stability and flocculation $\quad \mathbf{1 3}$

$\begin{array}{ll}\text { Chapter 3. Results and Discussion } & 15\end{array}$

$\begin{array}{ll}3.1 \text { Results } & 15\end{array}$

3.1.1 Zeta potential measurements of surfaces and particle suspensions $\mathbf{1 5}$

3.1.2 Zeta potential of microparticles in a nanoparticle dispersion 19

3.1.3 Microsphere stability and flocculation and the turbidity exponent 22

3.1.4 Microsphere stability experiments without added nanoparticles $\mathbf{2 3}$ 
References 


\section{List of Figures}

Figure 1. Zeta potential on fused silica slides as a function of $\mathrm{pH}$

Figure 2: Zeta potential vs. $\mathrm{pH}$ for the $1 \mu \mathrm{m}$ silica spheres

Figure 3: Zeta potentials of latex nanoparticles

19

Figure 4: Example fit of the UV/Vis spectrum of a single silica suspension

23

Figure 5: Turbidity curves of $0.1 \% 1 \mu \mathrm{m}$ silica microspheres, at varying $\mathrm{pH}$

Figure 6: Stability images of $0.1 \% \mathrm{v} 1 \mu \mathrm{m}$ silica microspheres

Figure 7: Turbidity curves of $0.1 \% \mathrm{v}$ silica microspheres in solution with $0.5 \% \mathrm{v}$ sulfate latex nanoparticles

Figure 8: Stability images of $0.1 \% \mathrm{v} 1 \mu \mathrm{m}$ silica microspheres with and without $0.5 \% \mathrm{v}$ sulfate latex nanoparticles

Figure 9: Turbidity curves for $0.1 \% \mathrm{v}$ silica with varying concentrations of sulfate latex nanoparticles, all at $\mathrm{pH} 2.5$

Figure 10: Stability images of $0.1 \% \mathrm{v} 1 \mu \mathrm{m}$ silica microspheres with and without $0.5 \% \mathrm{v}$ amidine latex nanoparticles.

Figure 11: Flocculation behavior of $0.1 \% \mathrm{v}$ silica microspheres in solution with $0.5 \% \mathrm{v}$ amidine latex nanoparticles

Figure 12a: SEM images of fused silica after immersion for $30 \mathrm{~min}$. in $0.1 \% \mathrm{v}$ sulfate latex, below $\mathrm{pH} 3.0$ 
Figure 12b: SEM images of fused silica after immersion for $30 \mathrm{~min}$. in $0.1 \% \mathrm{v}$ sulfate latex, above $\mathrm{pH} 3.0$

Figure 13: Surface density of adsorbed sulfate latex nanoparticles

Figure 14: Adsorption of sulfate latex particles on the $1 \mu \mathrm{m}$ silica spheres, with different amounts of time spent in the rinse solution (at $\mathrm{pH} 2.0$ )

Figure 15: Sulfate latex nanoparticle adsorption on silica at $\mathrm{pH}$ 2.0, varying concentration of nanoparticles

Figure 16: An example of contrasting regions of particle adsorption for $3.0 \% \mathrm{v}$ sulfate latex at $\mathrm{pH} 2.0$

Figure 17: Silica surfaces with adsorbed amidine latex ( $0.5 \% \mathrm{v}$ solution), varying $\mathrm{pH}$

Figure 18: Comparison of latex adsorption at $\mathrm{pH} 2.0$ on silica slides

Figure 19: Comparison of approximate amidine latex and sulfate latex particle densities

Figure 20: Nanoparticle patch size schematic

Figure 21: Probability density curves for the two types of nanoparticles used

\section{Tables}

Table 1: Zeta potential comparison 


\section{Chapter 1}

\section{$\underline{\text { Introduction }}$}

The stability of particles in solution is an important factor in any application that deals with colloidal suspensions. Solutions containing suspended micro-scale or nano-scale particles serve a wide variety of purposes. Colloids can be precursors to nanomaterials, composites, and other materials. They are used in inks, paints, and other emulsions, pharmaceutical products, and are found naturally in clays, biological fluids such as blood, natural organic matter colloids, and petroleum and geological processes. The stability of colloidal particles during transport through porous media is important in separation processes and the spread of contaminants, nutrients, and bio-solids through soil [1-10]. In all applications it is necessary to be able to maintain the colloid in a dispersed with the particles suspended as individual particles and with minimal amounts of flocculation.

The effects of additional material in a colloidal solution have been studied for decades, whether it is with the addition of polymer, polyelectrolyte, nanoparticles, or even microparticles [11-15]. One well documented effect of adding non-adsorbing nanoparticles or polyelectrolyte on colloidal stability is flocculation due to the "depletion" force, which has been observed and studied since the 1920s [12]. This force arises when the secondary particles or macromolecules in a solution are larger than the solvent molecules but significantly smaller than the suspended colloidal particles. As two colloidal particles move closer together, the concentration of the smaller particles surrounding the larger particles is altered. This creates a lower osmotic pressure relative to the bulk solution, which creates an attractive force between the larger colloidal particles $[14,16]$. 
The magnitude of the depletion force can be adjusted by controlling the concentration of smaller particles, while the range of the force can be adjusted by changing the size of the smaller particles relative to the larger particles. For example, depletion flocculation experiments using sodium polystyrene sulfonate (SPSS) as a non-adsorbing macromolecule in solution with colloidal polystyrene particles has been reported have been done by Walz and coworkers $[12$, 16]. Critical flocculation concentrations of SPSS in solutions of $473 \mathrm{~nm}$ colloidal polystyrene particles (at $0.01 \% \mathrm{v}$ ) reported to approximately $0.2 \% \mathrm{w}$ SPSS. Increasing the size of the polystyrene relative to the SPSS by using $960 \mathrm{~nm}$ polystyrene $(0.01 \% \mathrm{v})$ reduces the critical concentration of SPSS by about $50 \%$ to about $0.1 \% \mathrm{w}$ [16]. Similar experiments by Snowden and coworkers using colloidal silica have shown critical concentrations for depletion flocculation to be around $0.5 \% \mathrm{v}$. Additionally, bridging flocculation was observed in experiments where the polymer adsorption to the surface of the silica was favorable [17]. In the case of bridging flocculation, the polymer adsorbs to multiple particles, "bridging" the gap between and inducing flocculation.

Additional depletion studies by Walz and coworkers have shown the effects of silica nanoparticles on colloidal polystyrene $[12,16]$. Depletion flocculation occurs in $0.01 \% \mathrm{v}$ suspensions of polystyrene with silica concentrations of $2-3 \% \mathrm{v}$. The critical concentration is lower in systems with a higher size ratio of particles. Additionally, using higher concentrations of nanoparticles (beyond the critical flocculation concentration) can actually restabilize the polystyrene, shown through turbidity and flocculate size measurements. This restabilization is consistent with the force curve models for the systems, which show long range repulsive barriers that develop with increasing concentrations of nanoparticles. This restabilization is more prevalent with nanoparticles compared with polymers, which is attributed to the shape of the 
particles. The spherical nanoparticles produce the repulsive energy barriers much more quickly than the rod-shaped polymer, leading to restabilization.

In suspensions without depletion effects, stabilization of the colloidal particles is typically controlled through steric or electrostatic interactions between particles. Steric stabilization typically occurs through the use of polymer chains which are either adsorbed, grafted, or otherwise attached to the surface of the dispersed particles [18-22]. The polymer chains extend away from the particle surface and physically inhibit the particles from approaching other particles close enough to be in the range of the van der Waals attraction.

In order for polymers to be effective steric stabilizers, it is necessary for thickness the polymer layer to be at least on the order of the radius of the colloidal particles that the polymer coats, specifically for weakly coagulating organic dispersions. For other materials such as heavy metals, polymer layers thicknesses are required to significantly exceed the particle radius [22]. Additionally, the polymers must be sufficiently rigid and the layer sufficiently dense to achieve effective stabilization.

Block copolymers produce superior stabilization effects over homopolymers [21]. The most effective stabilization occurs when one block of the copolymer has a significantly higher affinity for adsorbing to the colloid surface. The length of the polymer and the ratio of the chain lengths of adsorbing and non-adsorbing blocks are important factors in determining the degree of adsorption. In the case of di-block copolymers, overall long chains with short but strongly adsorbing block and long non-adsorbing tails provide superior coverage and stabilization, comparable to that of grafted layers [21]. 
Ultimately, steric effects on stabilization are most effective when coupled with electrostatic repulsion. Colloids can be stabilized through the adsorption of additional charged polymer, such as polyacrylic acid (PAA) $[18,19]$. With colloidal titanium dioxide, experimental data shows that increasing the concentration of PAA in the supernatant leads to the zeta potential becoming more negative. As the molecular weight of the PAA was increased, the degree of adsorption increased (and zeta potential became even more negative), however this also enhanced the steric component of the repulsion between the particles by increasing the complexity of and the degree to which the polymer extended from the surface. This "electrosteric" stabilization was limited in that the molecular weight of the PAA could reach a critical value, a point at which the polymer began to cause bridging adsorption between particles, which decreased the overall adsorption and induced flocculation [19].

A more recent development in colloidal stability is through a nanoparticle-microparticle interaction described as "nanoparticle haloing" [7, 20, 23-30]. This behavior requires a system of two different types of particles that are highly different from each other in size and surface potential. Specifically, there must be larger weakly-charged micro-scale particles in solution with smaller nano-scale particles that have a comparatively large surface potential. The microscale particles alone have a weak enough charge that they exhibit flocculation due to van der Waals attraction. When highly charged nanoparticles are added to the microparticles at appropriate $\mathrm{pH}$, the nanoparticles organize around the microparticle surface, though due to their high charge there is sufficient repulsion between nanoparticles to prevent them from depositing on the microparticle itself. This forms the charged "halo" of nanoparticles around the microparticles, which effectively creates an electrostatic repulsive force between two "haloed" 
particles. The particles then remain stable in the same manner as highly charged microparticles alone, relying on the net charge to prevent flocculation.

The bulk of the work that has been done regarding nanoparticle halos has used silica microspheres as the weakly charged particle and either zirconia or polystyrene latex for the highly charged nanoparticles. Lewis and coworkers describe three scenarios in the silica microsphere-polystyrene latex nanoparticle system: a strongly attractive system with positively charged microparticles and negatively nanoparticles, where bridging flocculation occurs between critical nanoparticle volume fractions, a strongly repulsive system with both types of particles being highly negatively charged and depletion flocculation occurring above a critical nanoparticle volume fraction, and the haloing system with weakly charged microparticles and highly charged nanoparticles that exhibits stable behavior in the haloing range of volume fractions and flocculation elsewhere [27].

Modeling of the interaction potential in the weakly interacting case indicated that the nanoparticles encounter a small repulsive energy barrier, mere nanometers from the surface, which would be enough to prevent the particle from reaching the surface while remaining in very close proximity $[7,27,30]$. The observed stability in these conditions was quantified by measuring the sedimentation rate of the microparticles in solution relative to that of an individual particle. With nanoparticle volume fractions between $10^{-3}$ and $10^{-2}$, the particle velocity was effectively identical to that of a single particle, while outside that concentration range, the sedimentation velocity was around two orders of magnitude larger than that of an individual particle [27]. An increase in observed sedimentation velocity relative to the velocity of an individual particle implies that there has been an increase in effective particle size, which in these systems can only occur through aggregation of the microspheres. 
Measurements of the zeta potential of the microparticles show that in the strongly attractive case, there was a very large increase in zeta potential (approximately $90 \mathrm{mV}$ ), while in the weakly interacting and strongly repulsive case, there was only about a $14 \mathrm{mV}$ difference with and without nanoparticles [27]. This seems to indicate that in the weakly interacting solutions, the particles do not substantially adsorb to the silica, yet still provide stability in the suspension.

The sediments of the suspensions were analyzed with confocal microscopy to determine the packing structure of the particles $[24,27-29]$. In the stable haloing systems, the silica particles sediment individually, forming a polycrystalline, face-centered cubic structure in the sediment [27]. Additionally, the supernatant was analyzed after sedimentation, which revealed that the bulk nanoparticle concentration was relatively unchanged from the initial concentration. This indicates that there was negligible adsorption of the polystyrene onto the silica.

Experimental evidence of the haloing formation using zirconia nanoparticles was indicated by ultra small-angle x-ray scattering [26]. The results from these measurements indicate that zirconia particles remain at approximately $2 \mathrm{~nm}$ from the surface of silica spheres. This separation roughly corresponded to the Debye length of the silica. Additionally, it was reported that the distance between nanoparticles was significantly greater than the characteristic particle size.

Alternately, a very recent study has been done on polystyrene microspheres in suspension with zirconia nanoparticles in solution at $\mathrm{pH} 5$, a case where the zirconia nanoparticles were near their isoelectric point and the polystyrene were highly charged [31]. The results showed significant stabilization, based on the turbidity measurements as a function of time, with optimal nanoparticle concentrations of about $1.17 \%$. This stability appears to be produced as a result of 
adsorption of the zirconia on the polystyrene microspheres, based on scanning electron microscopy images of the particle surfaces.

The ability to maintain colloidal particles in a stable suspension is critical to their utility in all of the many potential applications of the particles. Colloidal stability can be achieved (or eliminated as in the cases of depletion flocculation) by the addition of nanoparticles or macromolecules and their interaction with larger colloidal particles. Stability studies involving depleting species as well as adsorbed or grafted stabilizing polymers have been well documented in the literature. Recent interest in nanoparticle-microparticle interactions and behavior, by nanoparticle haloing or adsorption, and the resulting studies show the possibilities for new methods for controlling the stability of colloids via tuning the electrostatics between particles. However, being a relatively new unexplored area of study, there is still much to be learned about these interactions and their potential for viable applications in colloidal applications.

The purpose of this project was to investigate the possibility of stabilizing weakly charged colloidal microspheres (silica) using highly charged nanoparticles (either sulfate polystyrene latex or amidine polystyrene latex, depending on whether a positive or negative particle charge is desired). The experimental techniques include determining the zeta potentials of the various particle species, both alone and in binary nanoparticle-microsphere solutions, measuring the flocculation rate and behavior of the particle solutions visually and with UV/Vis spectroscopy, and measuring and characterizing the degree of nanoparticle adsorption on silica surfaces, both visually with scanning electron microscopy and quantitatively with quartz crystal microbalance. In the remainder of this thesis, chapter 2 describes the materials and experimental methods that were used. Chapter 3 presents the results of the experiments as well as discussions on the results and chapter 4 provides the overall conclusions of the paper. 


\section{Chapter 2}

\section{Materials and Experimental Methods}

\subsection{Description of Materials}

Deionized water was used for all experiments. The water was produced by a RiOs 8 reverse osmosis system (Millipore, Billerica, MA, Catalog No. ZR0S6008Y) and followed by a Barnstead EASYpure II (Thermo Scientific, Waltham, MA, \# D7401) in series. Fused silica slides (Corning 7980 fused quartz silica, Item No. 3x1x1mm) were obtained from TGP (Technical Glass Products, Inc., Painesville, OH). The silica microspheres were supplied as a $10 \% \mathrm{w} / \mathrm{v}$ solution in water at a size specification of $1 \mu \mathrm{m}$ in diameter (reported mean diameter: $0.96 \mu \mathrm{m}$ ) by Polysciences, Inc. (Warrington, PA, Catalog No. 24326-15). The IDC (Interfacial Dynamics Corporation, Eugene, OR) brand polystyrene latex nanoparticles were supplied by Invitrogen Corporation (Carlsbad, CA). The sulfate latex nanoparticles (Catalog No. S37200) were supplied as an $8 \% \mathrm{w} / \mathrm{v}$ solution in water with a mean diameter of $0.022 \mu \mathrm{m}$. The amidine latex nanoparticles (Catalog No. A37309) were supplied as a $4 \% \mathrm{w} / \mathrm{v}$ in water with a mean diameter of $0.023 \mu \mathrm{m}$.

\subsection{Experimental procedures}

\subsubsection{Zeta potential measurements}

The zeta potential of the silica slides was determined using an SurPASS Electrokinetic Analyzer (Anton Paar GmbH, Graz, Austria). The SurPASS tubing was rinsed, using the extended rinse program instructions, with very dilute isopropanol ( $<1 \%$ by volume in water) and then with deionized water; rinse cycles were approximately $5 \mathrm{~min}$ for each. The fused silica 
microscope slides were cleaned with pure ethanol, rinsed in deionized water and cleaned for 30 min in a UV/Ozone ProCleaner (BioForce Nanosciences, Inc., Ames, IA). Once clean, the slides were mounted in the SurPASS clamping cell. A 5 min rinse cycle was then run using the chosen electrolyte, which had been adjusted to the highest $\mathrm{pH}$ for which that was to be measured using $0.2 \mathrm{M} \mathrm{NaOH}$. After rinsing, the measurement program was run, and zeta potential was measured at $\mathrm{pH}$ intervals from high to low $\mathrm{pH}$, being titrated using $0.2 \mathrm{M} \mathrm{HCl}$.

Measurements of the zeta potential of microspheres and nanoparticles (separately) were done using a Zetasizer Nano-ZS (Malvern Instruments Ltd, Worcestershire, UK), with samples measured in folded capillary cells (Part No. DTS1060C). To measure zeta potentials across a range of $\mathrm{pH}$ values, the Malvern MPT-2 Multi Purpose titrator was used, with $0.25 \mathrm{M} \mathrm{HCl}, 0.25$ $\mathrm{M} \mathrm{NaOH}$, and $0.01 \mathrm{M} \mathrm{HCl}$ as titrants.

To measure the effective microsphere zeta potentials in binary microsphere/nanoparticle solutions, a Micro-Electrophoresis Apparatus Mk II (Rank Brothers Ltd., Cambridge, England) was used. The instrument was set up in the rectangular cell configuration to avoid problems with sedimentation. Nanoparticle concentrations used with this instrument corresponded to those used in the flocculation and adsorption measurements, while the microsphere concentration was quite dilute $(<0.01 \% \mathrm{v})$ in order to be able to observe individual particles. All the measurements were taken at the stationary plane in the cell in order to eliminate the effects of the counter-flow of electrolyte in the cell. An electric potential was applied across the cell and the velocity of the particles was determined visually using a stopwatch to measure the time a particle took to travel a fixed distance. 
The stationary plane for the rectangular cell was calculated using Equation 1 (taken from the Rank Bros. instruction manual and corrected for the refractive indices of air and water). In Equation $1 s$ is the distance from the inner cell wall, $d$ is the cell depth, $h$ is the cell height, and $n_{a}$ and $n_{w}$ are the refractive indices of air and water, respectively.

$$
s=d\left[0.5-\left(0.0833+\frac{32 d}{\pi^{5} h}\right)^{0.5}\right] \frac{n_{a}}{n_{w}} \quad \text { Eq. } 1
$$

\subsubsection{ZetaSpin measurements}

Measurements of zeta potential on silica slides were attempted using the ZetaSpin rotating disk instrument. The instrument operates on the principle of generating radial flow outward from the center of a rotating disk, with the generated streaming potential measured between the center of the disk and the bulk solution [32]. Silica slides were cut into circles with 1 inch diameters and cleaned with ethanol before being cleaned in the UV/Ozone cleaner for 30 minutes.

The silica disks were then attached to 1 inch diameter rubber mounts, which in turn were mounted on the shaft of the instrument. The silica surface was immersed in the container of solution and positioned $1 \mathrm{~mm}$ above the center electrode; for the nanoparticle-free solution the test solution consisted of $1 \mathrm{mM} \mathrm{KCl}$ that was then titrated to various $\mathrm{pH}$ values. The maximum RPM was set to 4000 and $\mathrm{pH}$ and conductivity were measured using a handheld meter (Oakton 300 series, Oakton Instruments, Vernon Hills, IL). The zeta potential was measured from approximately $\mathrm{pH} 9.0$ to $\mathrm{pH} 2.0$. Solutions containing $1 \mathrm{mM} \mathrm{KCl}$ and $0.1 \% \mathrm{v}$ sulfate latex nanoparticles were used and measured at $\mathrm{pH}$ values in the same range. 
As explained in chapter 3, the experiments using ZetaSpin to measure the silica slide zeta potential were ultimately abandoned due to concerns over accuracy.

\subsubsection{Measurements of nanoparticle adsorption via scanning electron microscopy}

To evaluate the degree of nanoparticle adsorption on the silica surfaces, both fused silica slides and $1 \mu \mathrm{m}$ silica microspheres were prepared for viewing with scanning electron microscopy (SEM). The slides were first cleaned by sonication in ethanol (Bransonic 1200 Ultrasonic cleaner, Branson Ultrasonic Corporation, Danbury, CT). Initially, the slides were next cleaned via UV/Ozone, though once images showed no difference in behavior between surfaces with and without the UV/Ozone procedure, and also because colloidal silica spheres cannot be cleaned this way while in suspension, this step was later dropped. An appropriate volume of nanoparticle solution, at a given volume fraction, was prepared, briefly sonicated, and then titrated to the desired $\mathrm{pH}$ using $0.2 \mathrm{M} \mathrm{HCl}$ and measured using an Accumet Excel XL20 pH and conductivity meter (Fisher Scientific, Catalog No. 13-636-XL20). The nanoparticles used were, in separate experimental solutions, $0.02 \mu \mathrm{m}$ polystyrene latex with either sulfate surface groups and a negative surface potential or amidine surface groups for a positive surface potential.

A beaker of deionized water was then titrated, using $0.2 \mathrm{M} \mathrm{HCl}$, to the same $\mathrm{pH}$ as the nanoparticle solution. The cleaned and dried silica slides were briefly soaked in the $\mathrm{pH}$-adjusted water before being placed in a beaker containing the nanoparticle solution for $30 \mathrm{~min}$. After the adsorption time, the silica was removed from the latex particles and gently rinsed in the beaker of $\mathrm{pH}$-adjusted water in order to remove any excess (non-adsorbed) nanoparticles from the slide. By using the same $\mathrm{pH}$ in both solutions, the surface charge should remain the same and the 
adsorbed particles (if they were present) would remain on the slide. The silica was then allowed to air dry before the surfaces were imaged via SEM.

In order to prepare the silica microspheres for imaging, different methods were used initially. The first method was to allow the silica spheres to sediment completely while in a binary mixture with the latex nanoparticles, at a given $\mathrm{pH}$, in a cuvette. The resulting supernatant (containing only nanoparticles) was removed and replaced with nanoparticle-free water that was at the same $\mathrm{pH}$. The cuvette was inverted to redistribute the spheres, which were allowed to sediment again. This step was repeated for a total of three "rinse" cycles. After the final rinse, the spheres were allowed to sediment onto SEM sample mounts (cleaned with ethanol). The supernatant was removed, the samples air-dried, and then imaged via SEM.

It was suspected that there could be some desorption of nanoparticles off of the silica surfaces, as the spheres were exposed to the rinsing solution for a few hours (as it was required for the spheres to sediment entirely). Therefore, the procedure was modified to reduce the time that the silica spheres were exposed to the rinsing solution. The first method involved using only a single rinse of the particles, which reduced the exposure time to 2-3 hours. The second method involved speeding the sedimentation rate in a centrifuge at 12,000 RPM, which reduced the time exposed to a few minutes. The samples were then deposited on SEM mounts in the same manner.

The procedure that was then decided upon was to simply deposit the silica microspheres on a silica slide, which could then be immersed identically to the previous slides. A dilute solution of microspheres was titrated to the desired $\mathrm{pH}$ and a few drops of the solution were placed on the surface of a clean silica slide. The slides were allowed to air dry, which left a 
coating of spheres essentially adhered to the surface. The now-coated slides were rinsed in the $\mathrm{pH}$-adjusted water as before and again immersed in the nanoparticle solution for 30 minutes before being gently rinsed in the particle-free, $\mathrm{pH}$-adjusted water, and allowed to dry.

\subsubsection{Measurement of nanoparticle adsorption with quartz crystal microbalance}

Quartz crystal microbalance (QCM) measurements were done to evaluate the extent of nanoparticle adsorption on silica surfaces. The Q-Sense E4 quartz crystal microbalance with dissipation, or QCMD, was used with silica sensors (Q-Sense/Biolin Scientific, Linthicum, MD, Product No. QSX 303). The tubing and measurement cell were flushed with $2 \%$ v sodium dodecyl sulfate (SDS), and then flushed completely with deionized water. The sensors were sonicated for approximately 10 minutes in $2 \% \mathrm{v}$ SDS, rinsed thoroughly with deionized water, and air dried. The sensors were then cleaned in the UV/Ozone cleaner for 10 minutes. The clean sensors were loaded into the clean cells.

A reference solution containing deionized water that had been titrated to the desired $\mathrm{pH}$ was flowed slowly through the cell. Once the frequency of the sensor stabilized, $0.1 \% \mathrm{v}$ sulfate latex solution (titrated to the same $\mathrm{pH}$ as the reference solution) was introduced. The change in surface mass of the sensor was calculated from the change in the sensor frequency.

\subsubsection{Microsphere stability and flocculation}

To prepare samples for visual/photographic observation and UV/Vis spectroscopy, appropriate concentrations of latex nanoparticles (either amidine or sulfate) were prepared and 
titrated to the desired $\mathrm{pH}$ (using either $0.2 \mathrm{M} \mathrm{HCl}$ or $0.2 \mathrm{M} \mathrm{NaOH}$ ). For the microsphere-only samples, only deionized water was titrated, using the same titrants. The latex solution samples were sonicated for approximately 10 minutes, as were the undiluted $1 \mu \mathrm{m}$ silica microspheres. The titrated solutions were then transferred to cuvettes (in identical volumes per cuvette). The appropriate amount of microspheres was added, and the cuvettes capped and inverted in order to evenly distribute the particles. When using the UV/Vis spectrometer, the samples were all sonicated briefly a second time before the experiment, as there was more time in between the sample preparation and experiment.

The spectrometer used for the UV/Vis measurements was an Ocean Optics SD2000 miniature fiber optic spectrometer with an Ocean optics DT-1000-CE Deuterium Tungsten light source (Ocean Optics, Inc., Dunedin, FL). The absorbance spectrum was recorded at regular time intervals in order to calculate the turbidity parameter. Photographic experiments were carried out separately; the samples in cuvettes were lined up against a solid background while photos were taken every 5 minutes to observe any resulting flocculation or stabilization of the samples. 


\section{Chapter 3}

\section{$\underline{\text { Results and Discussion }}$}

\subsection{Results}

\subsubsection{Zeta potential measurements of surfaces and particle suspensions}

Initially, the zeta potential measurements on the flat silica surfaces were planned to be done using the ZetaSpin instrument. The goal was to be able to easily measure the zeta potential of the slide both in solutions containing nanoparticles, as well as nanoparticle-free solutions, in order to determine the extent to which any adsorbed nanoparticles would alter the effective zeta potential of the silica surface. This would have allowed for a simpler and more convenient measurement, compared to attempting to flow nanoparticle solutions through a parallel plate system, e.g. the Anton Paar SurPASS. The possibility of performing nanoparticle-silica slide zeta potential measurements with the SurPASS was decided against, as there were concerns over the impact of nanoparticles on the tubing and syringe pumps of the system, since it would be preferable to avoid contamination due to deposited particles.

The ZetaSpin, however, did not produce believable results for the zeta potential of the silica slide. The zeta potential vs. $\mathrm{pH}$ curve appeared to have the approximate curvature that was expected for silica, however the magnitude of the zeta potentials obtained were over $100 \mathrm{mV}$ larger than expected based on literature values [33]. Nonetheless, a few experiments were attempted using a $0.1 \% \mathrm{v}$ sulfate latex solution, with the thought that it still might be possible to measure the relative change in potential of the surface with and without nanoparticles. The measured potentials with the nanoparticles were not conclusively different from the silica-only 
potentials, so the ZetaSpin was used for no further experiments (data can be found in the Appendix A). Instead, the SurPASS was used for measurements on the silica slides.

Figure 1 shows the measured zeta potential of fused silica measured with $1 \mathrm{mM} \mathrm{KCl}$ as a background electrolyte. The data is compared with experimental data from the literature which was measured in a similar parallel plate configuration. The measured data indicates an isoelectric point (IEP) slightly below a $\mathrm{pH}$ of 2.0. The data reported in the literature used a linear regression to fit the portion of the data that trends upward toward the IEP. Based on this approximation, the data obtained using the SurPASS, and linearly extrapolating the data from the literature, results in very similar values for the IEP, at just under a $\mathrm{pH}$ of 2.0 [33].

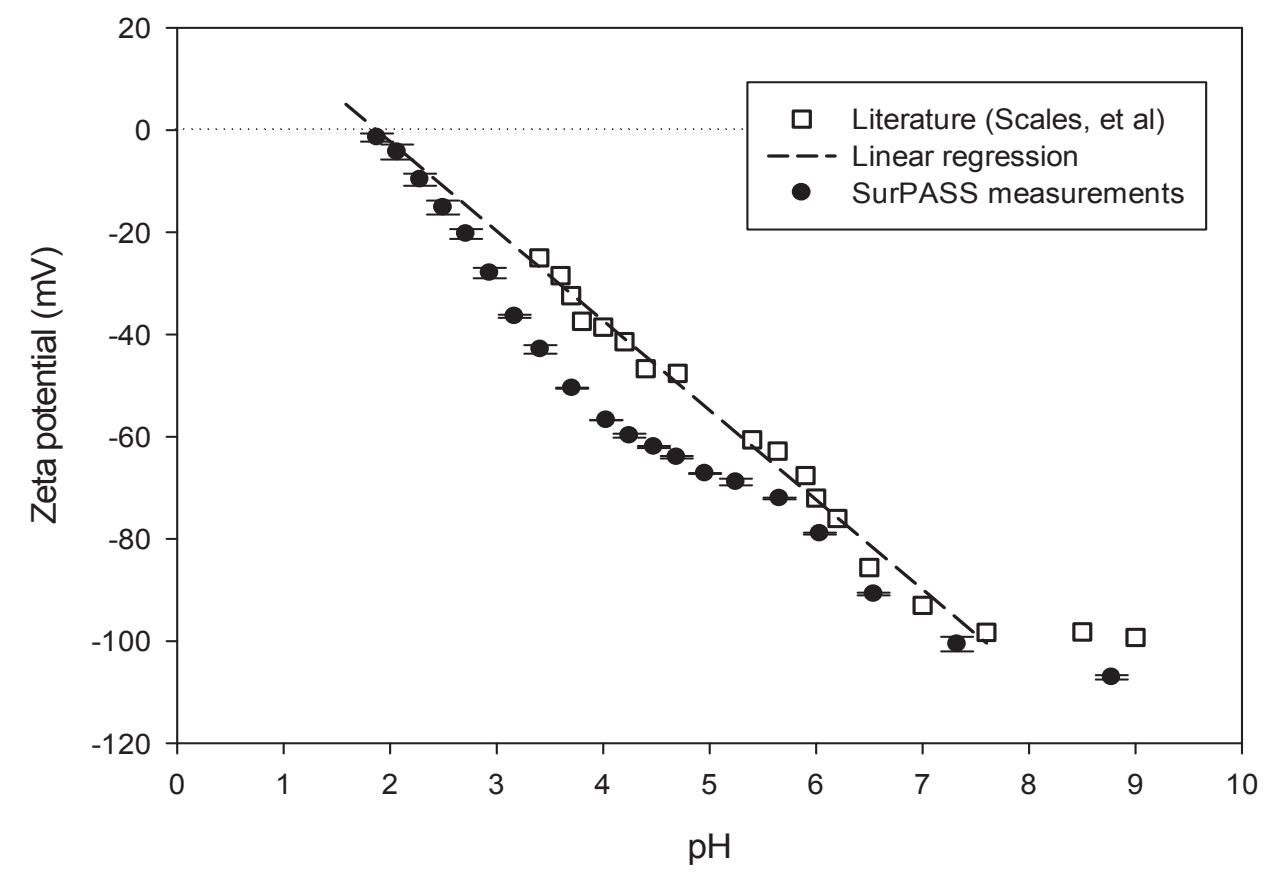

Figure 1. Zeta potential on fused silica slides as a function of $\mathrm{pH}$ with $1 \mathrm{mM} \mathrm{KCl}$ in water. Experimental data using the SurPASS (solid circles, with error bars indicating the standard deviation) is compared with literature data (open squares) [33]. 
The zeta potential of $1 \mu \mathrm{m}$ silica microspheres is shown in Figure 2, as measured using the Zetasizer. The potential of the microspheres is overall less negative than that of the fused silica slides. Both show highly negative zeta potentials that are relatively constant approximately above $\mathrm{pH} 7.0$, however the maximum potential is about $-100 \mathrm{mV}$ for the silica slides as compared to about $-75 \mathrm{mV}$ for the microspheres. The IEP occurs at approximately $\mathrm{pH}$ 3.0.

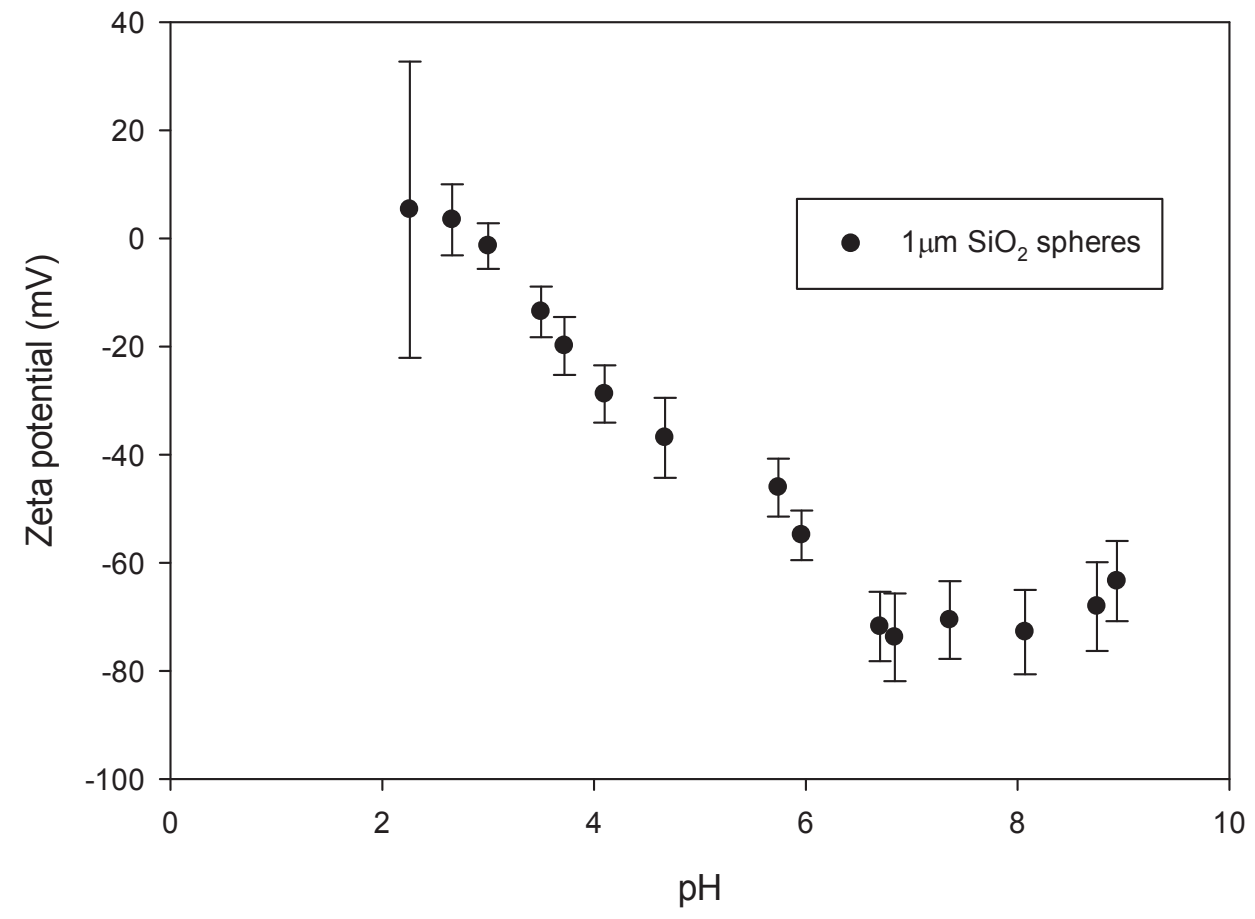

Figure 2: Zeta potential vs. $\mathrm{pH}$ for the $1 \mu \mathrm{m}$ silica spheres. Standard deviation is indicated by the error bars.

Figure 3 shows the measured zeta potentials for the polystyrene latex nanoparticles. Both types of nanoparticles, sulfate latex and amidine latex, remain relatively constant over the $\mathrm{pH}$ range that was used. The sulfate particles are negatively charged, with a zeta potential between 
approximately -60 to $-70 \mathrm{mV}$ above $\mathrm{pH}$ of 3.0 ; as the $\mathrm{pH}$ approaches 2.0 and lower, the potential begins to increase slightly (decrease in magnitude). The amidine particles are effectively the opposite. The zeta potential is between about +65 and $+75 \mathrm{mV}$ above about $\mathrm{pH} 3.0$, after which the potential decreases slightly. There is some variability between measured points, and at a given $\mathrm{pH}$ point the measured potential seems to vary in a range of about $10 \mathrm{mV}$.

The standard deviation in each measurement is quite large relative to the magnitude of the zeta potential. The particle size (approximately $20 \mathrm{~nm}$ ) is towards the lower limit of the suitable range for zeta potential measurements (using the Zetasizer), so it is possible that there is more inherent error in the measurement than there is with larger particles. However, the average zeta potentials were consistent over multiple measurements. This suggests that the deviation arises not from the measurements, but is a actual indication of the deviation in zeta potential between different particles. 


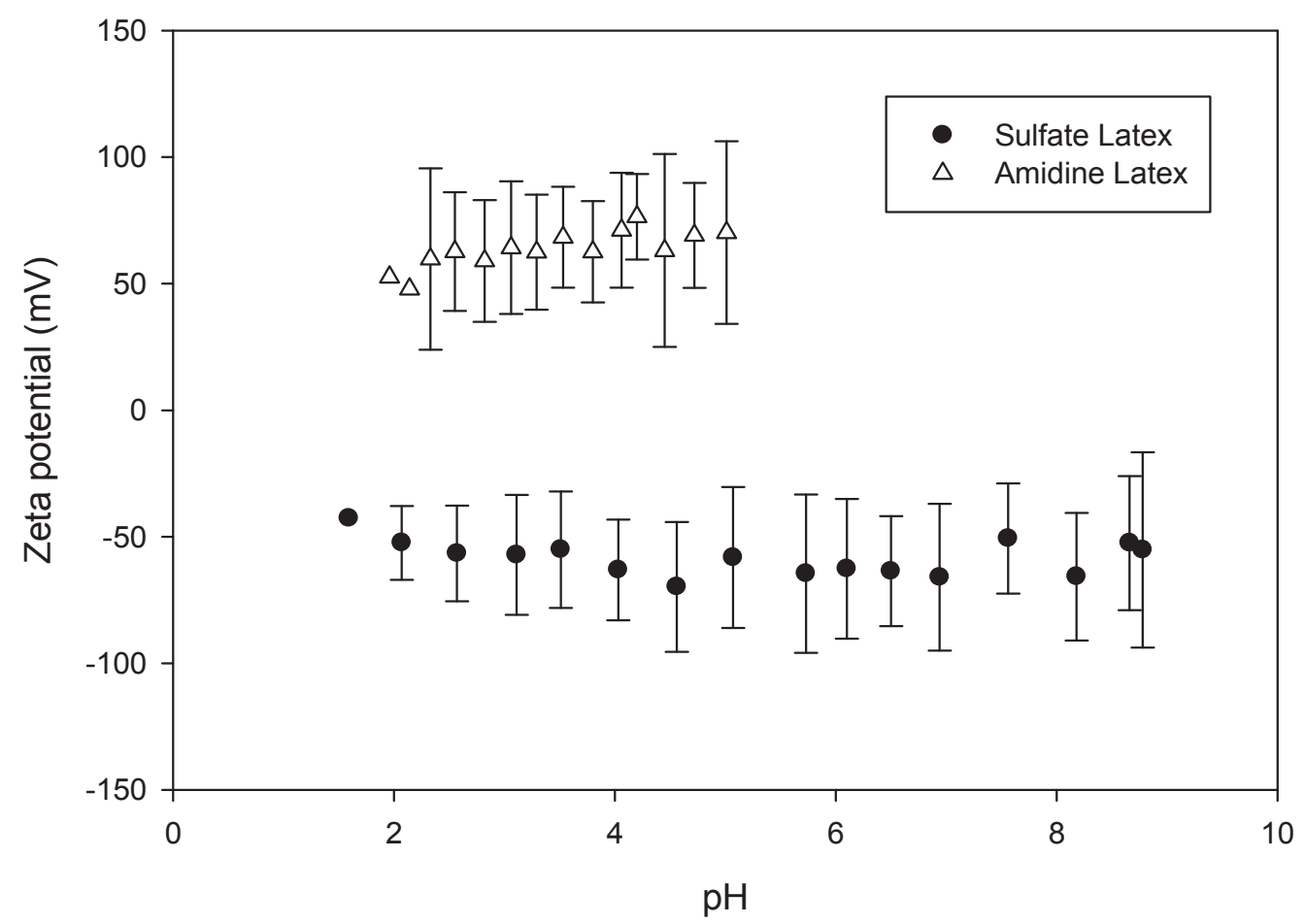

Figure 3: Zeta potentials of sulfate latex (circles) and amidine latex (triangles) nanoparticles. The standard deviation is indicated by the error bars.

\subsubsection{Zeta potential of microparticles in a nanoparticle dispersion}

A separate method for determining whether the nanoparticles deposit onto the larger silica microspheres is to measure the zeta potential of the microspheres in solutions with and without added nanoparticles. Because of the vast difference in zeta potential of the two species, specifically at $\mathrm{pH}$ vales near the isoelectric point of silica, nanoparticle deposition should result in a noticeable change in the electrophoretic mobility of the silica. This method of determining the zeta potential has been used for many years $[34,35]$. The Rank Brothers micro- 
electrophoresis apparatus was used to do this measurement, as it allowed for focusing on the silica microspheres alone without measuring the zeta potential of any free nanoparticles.

Using the Rank Brothers micro-electrophoresis instrument, the applied electric field (Equation 2) and the particle velocity were used to calculate the electrophoretic mobility (Equation 3), which was used to calculate the zeta potential via the Smoluchowski equation (Equation 4). $E$ is the electric field, $V$ is the applied voltage, $R$ is the cell resistance (found by measuring the voltage and current in the cell and using Ohm's law), $\mu_{\mathrm{E}}$ is the electrophoretic mobility, $v$ is the particle velocity, $\zeta$ is the zeta potential, $\eta$ is the solution viscosity, $\varepsilon$ is the permittivity of the solution.

$$
\begin{array}{lc}
E=\frac{V}{R K A} & \text { Eq. } 2 \\
\mu_{E}=\frac{v}{E} & \text { Eq. } 3 \\
\zeta=\frac{\eta \mu_{E}}{\epsilon} & \text { Eq. } 4
\end{array}
$$

Table 1 shows the effective composite zeta potential for the binary mixtures of silica and polystyrene particles. The measurements were done at $\mathrm{pH} 2.0$ and 3.0 using the Rank Brothers Micro-Electrophoresis Apparatus Mk II. The values for the silica only samples are more approximate than for the binary solutions since in both cases the silica spheres were very weakly charged and the particles were moving very slowly (sometimes essentially stationary), so it was more difficult to get an accurate measurement of the zeta potential. Zeta potential measurements of pure $1 \mu \mathrm{m}$ silica spheres using the Zetasizer (Figure 2) are included in the table for comparison. 
There is a very significant difference between the zeta potential of the silica alone compared with the binary mixtures of silica and polystyrene latex nanoparticles. There is no doubt that the spheres are much more highly charged in the solutions containing the nanoparticles. Determining the zeta potentials using the Micro-Electrophoresis Apparatus Mk II allows for direct visual observation of the particle movement in solution. The silica-only samples were barely moving, taking minutes to cross the field of view, while the silica with added nanoparticles crossed the field of view in a matter of seconds. Both the sulfate latex and amidine latex result in the silica spheres effectively becoming quite highly charged at low $\mathrm{pH}$. For both binary solutions the absolute magnitude of the potential is similar to, or even greater than, the potential observed a pH 6 and greater for silica-only solutions.

\begin{tabular}{|c|c|c|}
\hline & $\mathrm{pH} 2.0$ & pH 3.0 \\
\hline Silica only & +7.3 & +2.1 \\
\hline Sulfate latex only & -52 & -57 \\
\hline Silica with $0.5 \%$ v Sulfate latex & -63.2 & -108.1 \\
\hline Amidine latex only & +53 & +64 \\
\hline Silica with $0.5 \% \mathrm{v}$ Amidine latex & +79.6 & +83.1 \\
\hline
\end{tabular}

Table 1: Comparison of composite zeta potentials $(\mathrm{mV})$ for $1 \mu \mathrm{m}$ silica microspheres and polystyrene latex nanoparticles.

The effective zeta potential of silica with adsorbed nanoparticles is shown in Table 1 to be greater than that of the nanoparticles alone. This could be explained by the nature of the zeta potential measurement, which measures the potential at the slipping plane. The adsorbed 
nanoparticles create a complex surface with locally varying curvature. Because the relationship between electrophoretic mobility and zeta potential depends on the radius of curvature, accurate determination of the zeta potential for particles with adsorbed nanoparticles is non-trivial. For this reason, the values reported for microsphere/nanoparticle mixtures in Table 1 should be viewed as rough estimates of the zeta potential.

\subsubsection{Microsphere stability and flocculation and the turbidity exponent}

Optical turbidity measurements were used to quantitatively measure the rate at which the samples flocculated. The approach involves measuring the total absorbance in a UV/Vis spectrophotometer and then calculating a turbidity exponent, $n$, that depends on the size of the scattering particles. To calculate the turbidity of the particle solutions, the absorbance spectrum from 500-700 nm (wavelength) was used. The data was fit to Equation 6 [36] to calculate the turbidity exponent, $n$. Equation 6 is derived from the relationship between the intensity of light scattered via Rayleigh scattering and wavelength $\lambda$, which depends on $\lambda^{-4}$. This relationship only applies to particles that are much smaller than the wavelength of the scattered light. For particle sizes significantly greater than $\lambda$ (i.e., Debye scattering, which applies to the $1 \mu \mathrm{m}$ silica spheres used in these experiments), the -4 exponent becomes the variable, $n$. As particle size increases in a system, the exponent $n$ in Equation 5 has been shown experimentally to become less negative $[17,36,37] . A$ is the absorbance (specific turbidity) $[12,16,37]$ at wavelength $\lambda, k$ is a constant which depends on particle size, density, and refractive index, and $n$ is the turbidity exponent.

$$
\begin{gathered}
A=k \lambda^{n} \\
\ln (A)=n \ln (\lambda)+\ln (k) \quad \text { Eq. } 5 \\
\text { Eq. } 6
\end{gathered}
$$


The data fitting was done by plotting $\ln (A)$ vs. $\ln (\lambda)$, fitting a straight line to the curve, with $n$ as the slope (Equation 6). Figure 4 shows an example of the linearly fitted data, plotted as $\ln (A)$ vs. $\ln (\lambda)$ of a flocculating silica microsphere solution at both 0 and 20 minutes, which shows the slope of the fit becoming less negative as the flocculation progresses.

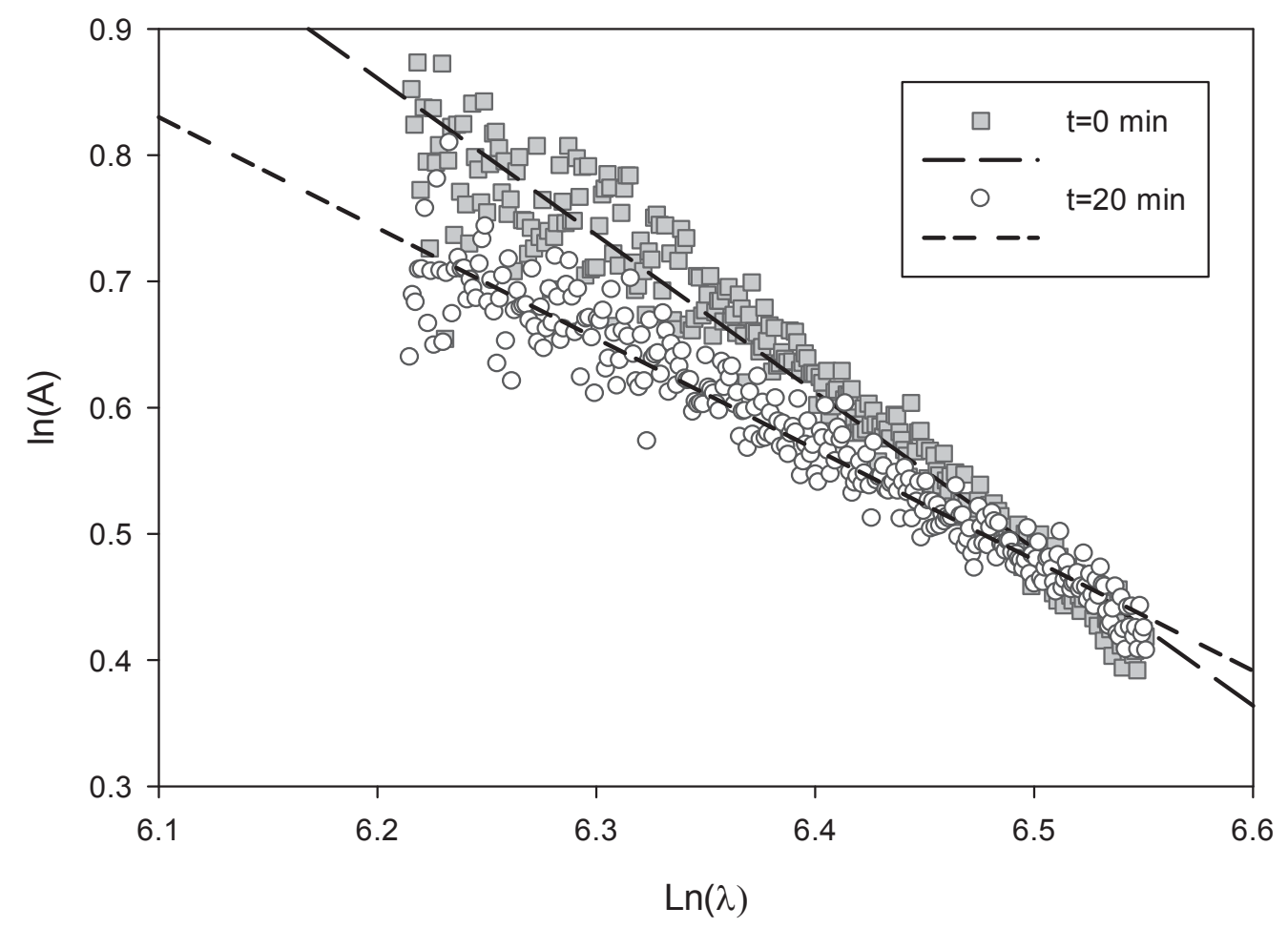

Figure 4: Example fit of the UV/Vis spectrum of a single silica suspension at two separate times. The dashed lines are the linear fit of the data.

\subsubsection{Microsphere stability experiments without added nanoparticles}

Figure 5 shows the resulting turbidity curves for solutions containing only $0.1 \% \mathrm{v} 1 \mu \mathrm{m}$ silica microspheres. The $\mathrm{pH}$ ranged from $\mathrm{pH} 2.0$ to 8.2. As particle size increases, the turbidity 
exponent becomes less negative. From the data, there is a clear difference between samples at $\mathrm{pH} 4.0$ and lower, compared to 5.0 and higher. From about a $\mathrm{pH}$ of 5.0 and higher, the microspheres are effectively stable. The particle size, as gauged by the turbidity exponent, remains approximately unchanged over a period of two hours, while the lower $\mathrm{pH}$ solutions show a rapidly increasing particle size, with significant flocculation being visible almost immediately.

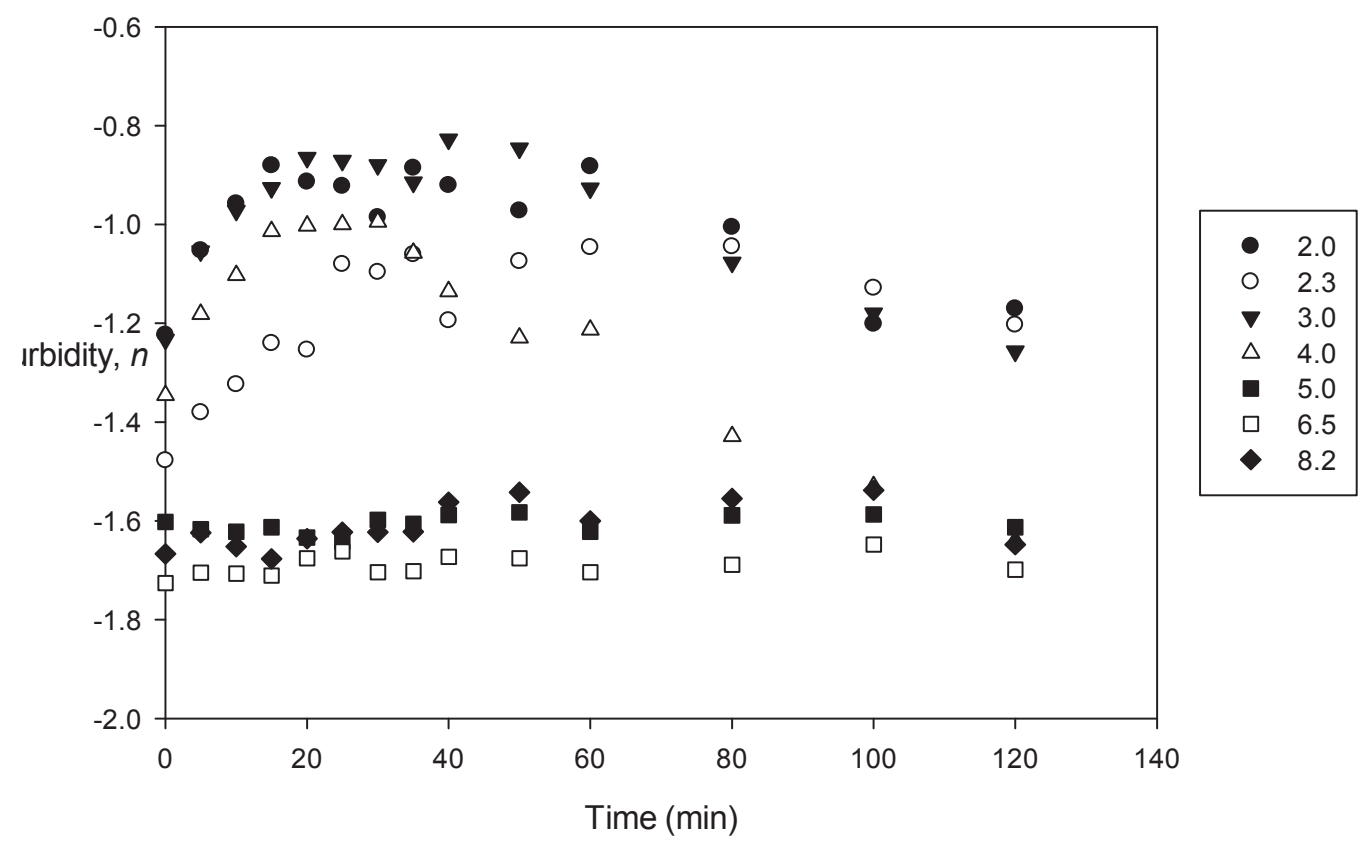

Figure 5: Turbidity curves of $0.1 \% 1 \mu \mathrm{m}$ silica microspheres, at varying $\mathrm{pH}$. The $\mathrm{pH}$ values used are labeled in the legend.

For the purposes of the plots of turbidity exponents with respect to time, the first measurement is taken to be effectively $t=0 \mathrm{~min}$. In actuality, however, there was a short interval on the order of 10 minutes between the introduction of the microspheres into the solution and the 
beginning of the measurement process. The samples were all sonicated again briefly right before the measurements, however it was nearly impossible to begin the measurements before the particles began flocculating. Since the flocculation process is quite rapid when it was observed (at low $\mathrm{pH}$ ), the particles had already begun to aggregate before $\mathrm{t}=0$ minutes, resulting in the different starting values for $n$ for the first measured point.

As the cuvettes of solution were not turned to redistribute particles during the time that the solutions were monitored, flocculating samples can exhibit a peak in the turbidity exponent, as the large flocculates that form initially sediment out of solution. This leaves smaller flocculates and individual particles still in suspension, which causes the turbidity exponent to start decreasing after reaching a peak value. Using the peak in the $n$-vs.-time curve as an indicator, the spheres flocculate and a significant amount of flocculates have sedimented after only 20 minutes.

Photographs of the flocculating silica-only solutions are shown in Figure 6. Solutions that are stable are cloudy due to scattering of light by the suspended microspheres. The lower $\mathrm{pH}$ solutions clearly flocculate and most of the particles sediment within an hour. The $\mathrm{pH} 4.0$ solution is somewhat in between the two extremes, as flocculation is visible but much slower than $\mathrm{pH}$ values that are slightly lower. At $\mathrm{pH} 5.0$ and higher, there is a very clearly defined boundary between the sedimenting spheres and the supernatant, indicating the spheres are sedimenting individually and uniformly. 


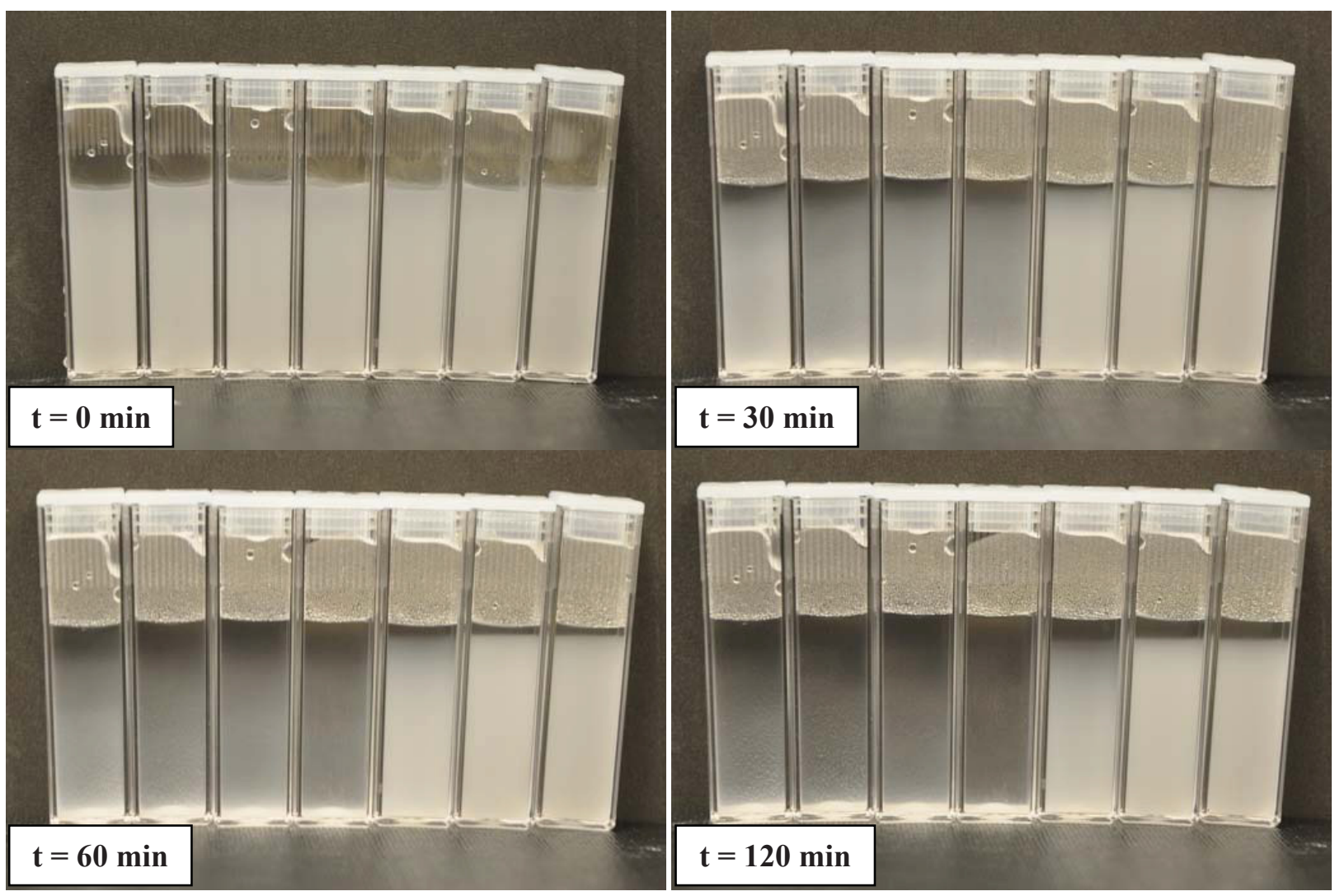

Figure 6: Stability of $0.1 \% \mathrm{v} 1 \mu \mathrm{m}$ silica microspheres. In each photograph, the cuvettes contain solutions at $\mathrm{pH}$ (L to $\mathrm{R}$ ) of 2.0, 2.5, 3.0, 3.5, 4.0, 5.0, and 8.0.

\subsubsection{Microsphere stability experiments with added nanoparticles}

The stability of $0.1 \% \mathrm{v}$ silica microspheres remains effectively unaffected upon the addition of $0.5 \% \mathrm{v}$ sulfate latex nanoparticles. The turbidity vs. $\mathrm{pH}$ curve is shown in Figure 7. This concentration of nanoparticles was initially chosen as it is in the range (approximately $0.1 \% \mathrm{v}$ to $1.0 \% \mathrm{v})$ suggested in the literature that would potentially exhibit a stabilizing effect [25, 27]. These authors suggested that it was possible to form "halos" of nanoparticles around microsphere surfaces. The key claim of the studies is that in cases where the microspheres and the nanoparticles interact weakly, but still repulsively, the particles concentrate a short distance 
from the silica surface, rather than truly adsorb. In doing so, the highly charged "halo" serves to stabilize the weakly charged microspheres, resulting in slow sedimentation of individual particles instead of flocculation and rapid sedimentation.

However, the microsphere/nanoparticle solutions studied in Figure 7 behave almost identically to the microsphere-only measurements (Figure 5), exhibiting the same characteristic rapid change in turbidity at low $\mathrm{pH}$ values. Indeed, based solely on the turbidity values, it appears that the microspheres may actually flocculate slightly faster or form larger aggregates, as well as more uniformly with the addition of nanoparticles when compared to Figure 5. Specifically, the peak in turbidity is approximately -0.7 for $\mathrm{pH}$ from $2.0-3.0$ with the added sulfate latex nanoparticles, whereas without the nanoparticles the peaks were between about -0.8 and -0.9. This difference could also result from a slight depletion attraction between the microspheres produced by the nanoparticles in solution, though this is not certain. Regardless, the important finding from the sulfate latex and silica systems is that the stability of the microspheres is clearly not improved. 


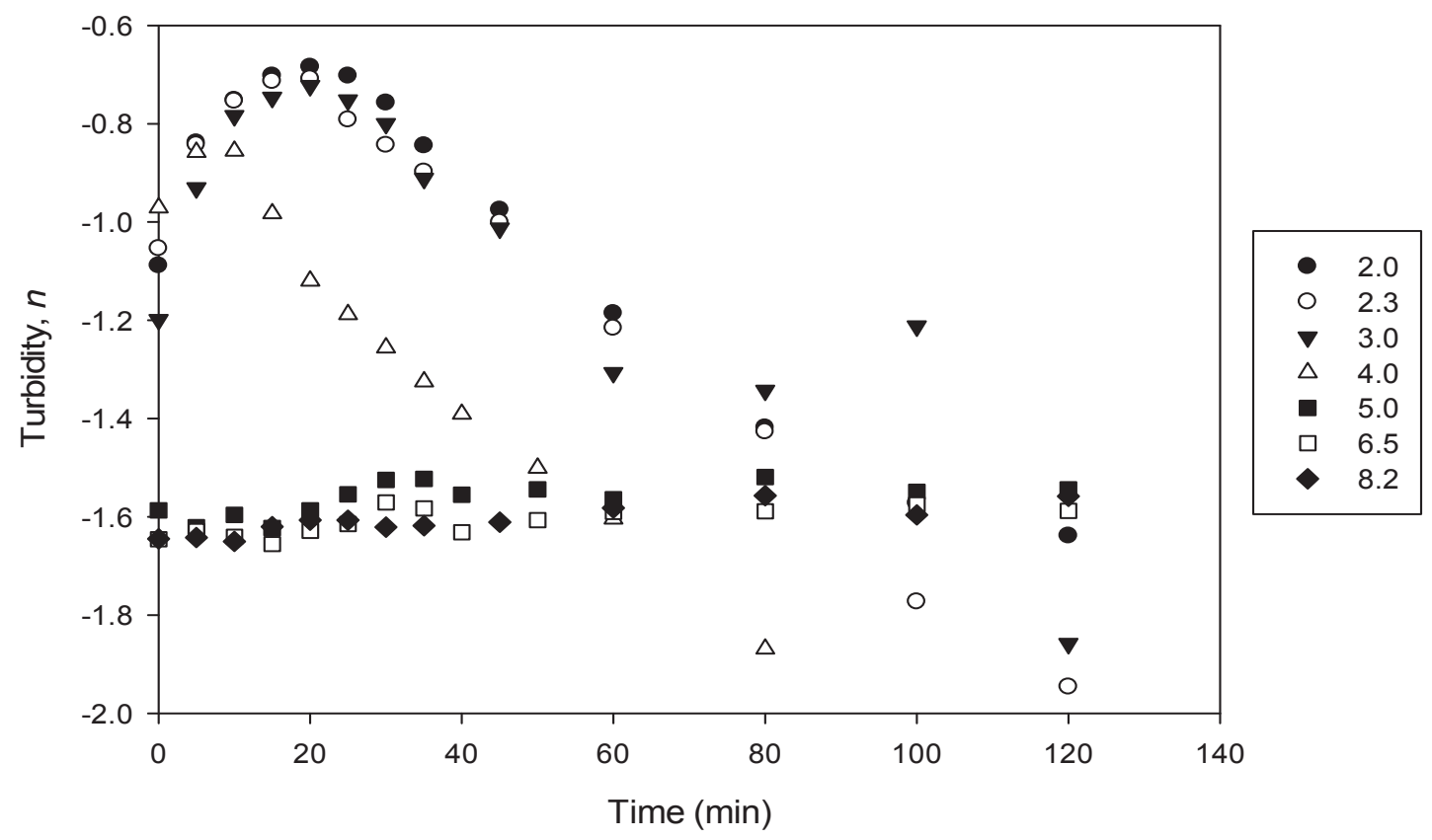

Figure 7: Turbidity curves of $0.1 \% \mathrm{v}$ silica microspheres in solution with $0.5 \% \mathrm{v}$ sulfate latex nanoparticles. The $\mathrm{pH}$ values used are labeled in the legend.

Figure 8 shows photographs of silica solutions at low $\mathrm{pH}$ values with and without the addition of sulfate latex nanoparticles. The silica-only samples flocculate as usual, and the samples containing the sulfate latex do as well. However, in this instance the $\mathrm{pH} 2.0$ sample is shown to flocculate slower than the rest. It still lacks as well of a defined boundary between the sedimenting particles and the remaining solution, however there are clearly more particles in suspension after 2 hours than the other cuvettes at the different $\mathrm{pH}$ values, and differs from the behavior of the pH 2.0 sample in Figure 7.

It is important to note that solutions containing the latex nanoparticles (both sulfate and amidine, presented below) exhibit a bluish color. When the solutions contain suspended silica particles as well, the color is less noticeable, however as the silica flocculates or sediments, the 
remaining supernatant is distinctly bluish in color when compared to the silica-only solutions. In Figure 8 as well as Figure 9, the focus should be on the opacity of the solution and the interface between the uppermost silica particles and supernatant. Non-flocculated samples will appear white in all cases. With this in mind while looking Figure 8, every cuvette except the silica/latex at $\mathrm{pH} 2.0$ is nearly transparent, indicating almost complete flocculation and sedimentation by the final image $(120 \mathrm{~min})$. The silica/latex solution at $\mathrm{pH} 2.0$ is noticeably bluish with a less-defined sedimentation boundary, as some degree of flocculation has occurred, allowing the bluish nanoparticle-containing supernatant to being to be visible.
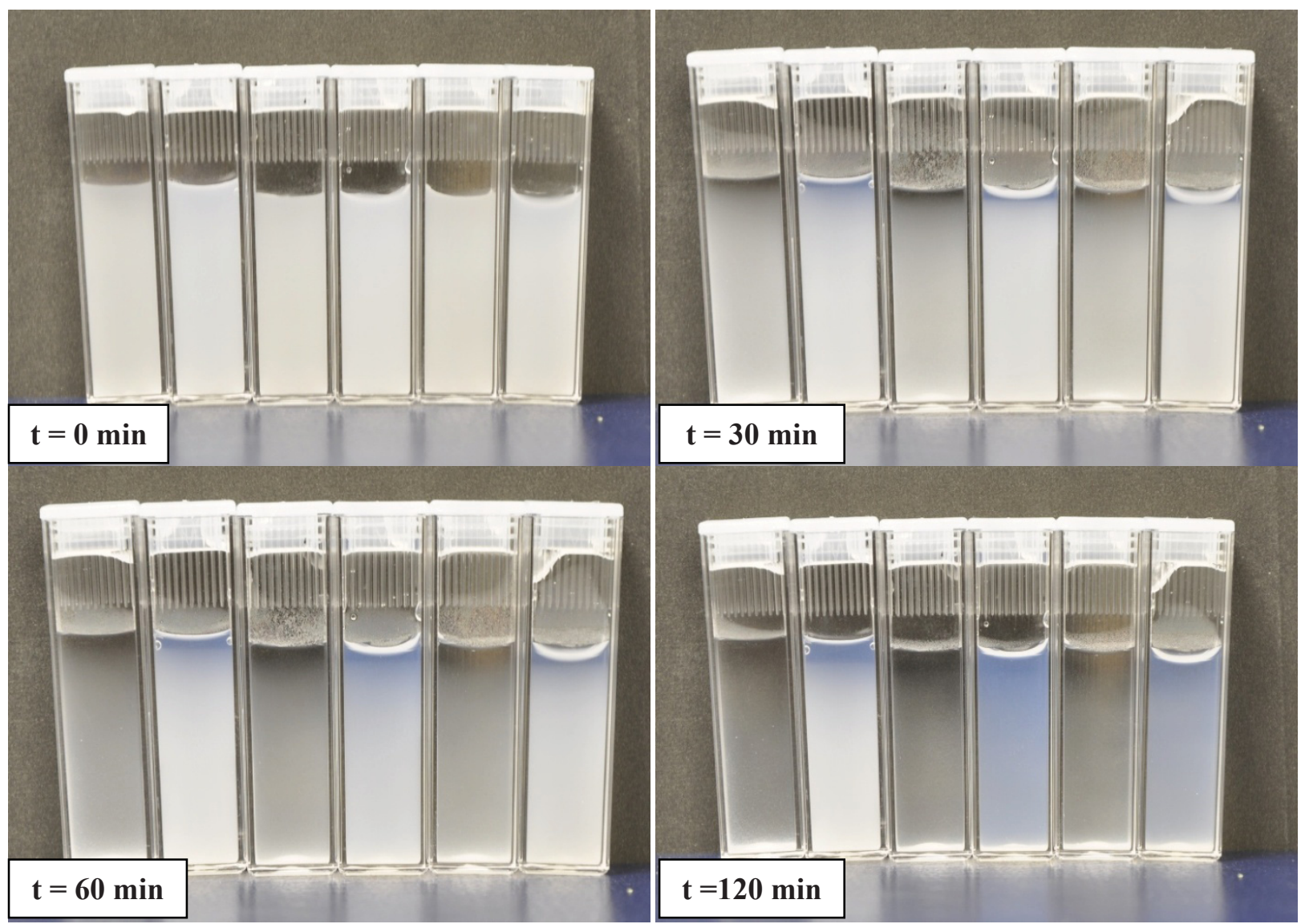

Figure 8: $0.1 \% \mathrm{v} 1 \mu \mathrm{m}$ silica microspheres with and without $0.5 \% \mathrm{v}$ sulfate latex nanoparticles. In the cuvettes from L to R: pH $2.0 \mathrm{SiO} 2$ only, $\mathrm{pH} 2.0 \mathrm{SiO} 2$ + latex, $\mathrm{pH} 2.5 \mathrm{SiO} 2$ only, $\mathrm{pH} 2.5$ $\mathrm{SiO} 2$ + latex, $\mathrm{pH} 3.0 \mathrm{SiO} 2$ only, $\mathrm{pH} 3.0 \mathrm{SiO} 2$ + latex. 
The behavior at $\mathrm{pH} 2.0$ seems somewhat contradictory toward the UV/Vis measurements. This is somewhat of an anomaly that was observed occasionally with other samples at varying $\mathrm{pH}$ values, both with and without nanoparticles. Previous trial experiments occasionally produced a sample that did not flocculate on one occasion, but it flocculated other times with no apparent change in sample conditions or preparation. For example, a silica-only sample was once observed to be semi stable at $\mathrm{pH} 3.0$, while samples at $\mathrm{pH}$ values directly above and this value flocculated. Furthermore, when the measurements for Figure 7 were being done, the sample visually appeared to be flocculating and sedimenting virtually identically to the other low $\mathrm{pH}$ samples. This indicates that the samples may be in conditions where the particles are close to a "border" between unstable and stable. Thus whether the particles flocculate or remain stable could result from very small changes in parameters that were not precisely controlled. Such variables include the inherent surface charge density of the particles, the sample age, the solution temperature, or even the degree of mixing.

Additionally, the UV/Vis spectrometry may be much more sensitive to flocculation than either simple visual or photographic observation. The photographs are purely macroscopic and rely solely on human observation, and flocculation only becomes visible once the larger aggregates begin to sediment, thus it may be possible for the spectrometer to register changes in the particle size before this becomes visible to the naked eye.

Another possibility for differences between measurements is that the sulfate latex nanoparticles used in the photographs were from a more recent batch than those that were used for the UV/Vis experiments. It is possible that slight differences in batches resulted in slight differences in ionic strength of the solutions or in the surface charge density of the nanoparticles. In situations where the particles may be on the brink of stability, changes in the solution could 
have a more pronounced effect. If one solution in fact had a slightly lower ionic strength than another, it would exhibit less screening of surface charges, and any nanoparticles that adsorbed to the silica surface would have a stronger effect on the effective charge of the particle, leading to a semi-stable solution.

Since a nanoparticle concentration of $0.5 \% \mathrm{v}$ was unable to sufficiently stabilize the silica at any observed $\mathrm{pH}$, the concentration was adjusted between $0.1 \% \mathrm{v}$ and $3 \% \mathrm{v}$ at $\mathrm{pH} 2.5$. Figure 9 compares the turbidity curves from $0-1.0 \% \mathrm{v}$. As seen, the turbidity exponent showed the same initial increase as observed in samples without added nanoparticles (Figure 5), meaning that the solutions flocculated again. The $3 \% \mathrm{v}$ sample flocculated as well, but this was only observed visually, not measured via the UV/Vis. It should be noted that turbidity curves for $0.1 \% \mathrm{v}$ nanoparticles do not exhibit quite the same peak as observed in the higher concentrations, and appears to flocculate slightly slower. 


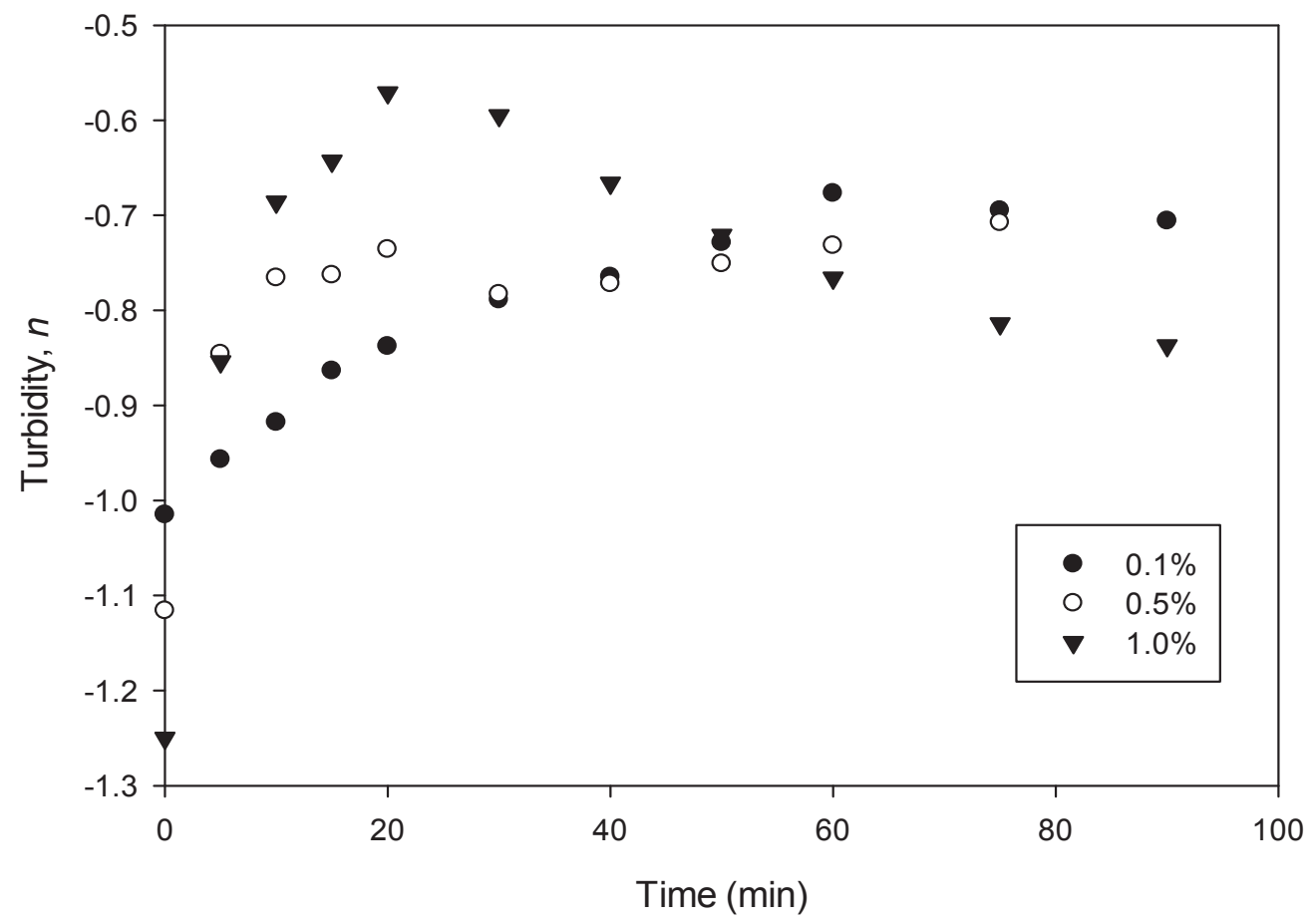

Figure 9: Turbidity curves for $0.1 \% \mathrm{v}$ silica with varying concentrations of sulfate latex nanoparticles, all at $\mathrm{pH} 2.5$. The legend indicates the nanoparticle concentrations that were used.

Figure 10 shows the same experimental tests as Figure 8, though with positively-charged amidine latex nanoparticles instead of the negatively-charged sulfate latex. Here, at all three $\mathrm{pH}$ values, the amidine latex very clearly stabilized the microspheres. Each solution has a well defined sedimentation boundary layer, just as observed with the stable, silica-only solutions in Figure 6. Thus there is a very distinct stabilization effect of using the positive amidine compared to the negative sulfate nanoparticles. This is somewhat unexpected, as for the three $\mathrm{pH}$ values show in Figure 10, the zeta potentials for the silica microspheres are all either effectively zero at $\mathrm{pH} 3$, or weakly positive at 2 and 2.5. This means the microspheres and nanoparticles are both positively charged (though the charge of the silica is still weak), and yet still result in a stable 
solution, while the analogous system with the sulfate latex (negative latex and weakly negative silica, at $\mathrm{pH}$ values on the other side of the IEP) was unstable.

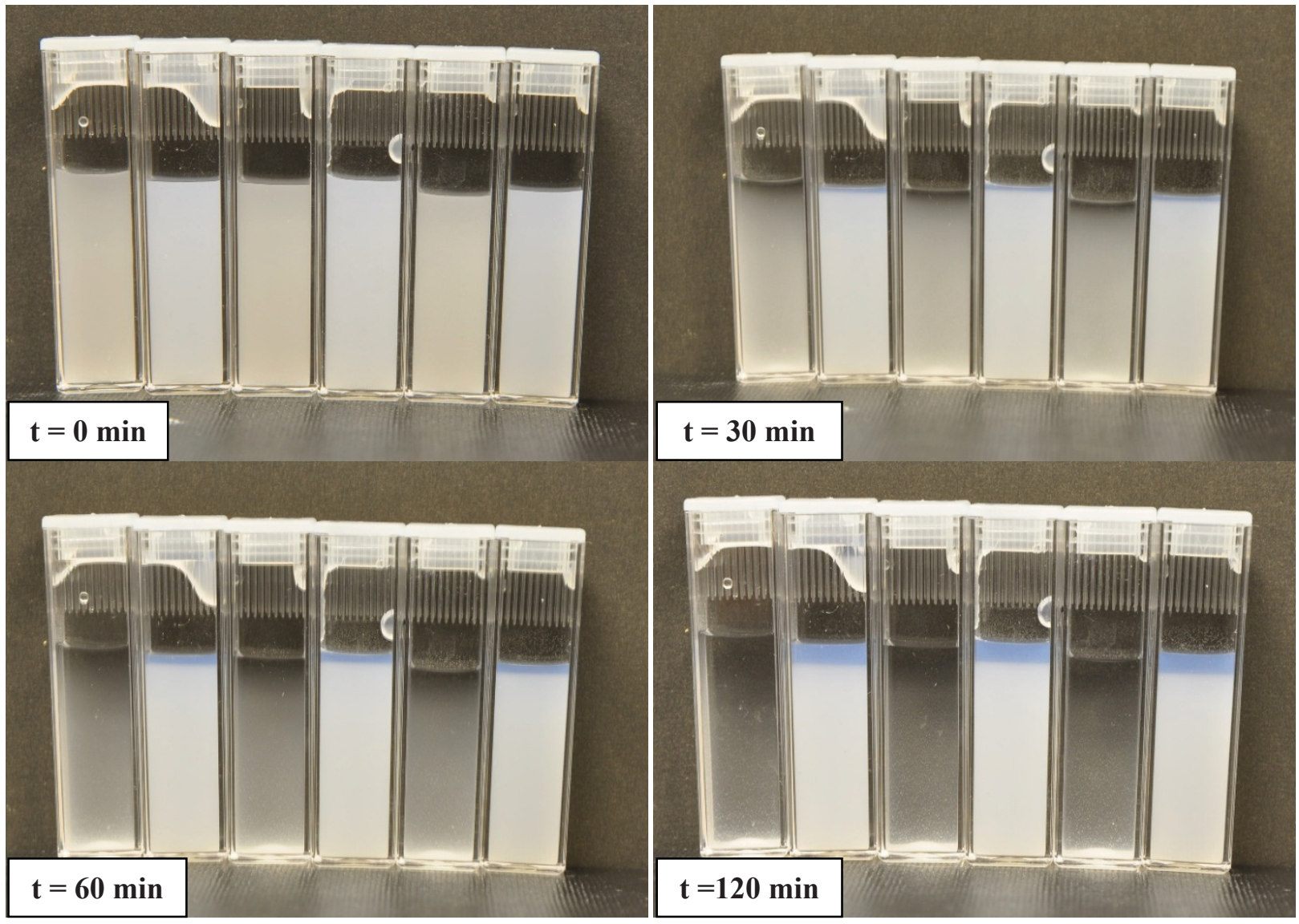

Figure 10: $0.1 \% \mathrm{v} 1 \mu \mathrm{m}$ silica microspheres with and without $0.5 \% \mathrm{v}$ amidine latex nanoparticles.

In the cuvettes from L to R: pH 2.0 SiO2 only, $\mathrm{pH} 2.0 \mathrm{SiO} 2$ + latex, $\mathrm{pH} 2.5 \mathrm{SiO} 2$ only, $\mathrm{pH} 2.5$ $\mathrm{SiO} 2$ + latex, pH $3.0 \mathrm{SiO} 2$ only, pH $3.0 \mathrm{SiO} 2$ + latex.

Figure 11 shows the turbidity exponents for amidine latex $(0.5 \% \mathrm{v})$ and $1 \mu \mathrm{m}$ silica $(0.1 \% \mathrm{v})$ at various $\mathrm{pH}$ values over the course of about 2 hours. As expected, based on what was observed in Figure 10, the turbidity exponents at each $\mathrm{pH}$ remain effectively the same over the course of two hours. The different samples had somewhat varying starting values, but they 
remained effectively constant throughout the entire time, with the turbidity exhibiting no discernable drift or curve either in the positive or negative direction.

The slightly different starting values across the $\mathrm{pH}$ range could result from the fact that the $\mathrm{pH}$ was adjusted after the particle suspension was prepared. The final volumes could have differed by a couple of percent due to the different amounts of titrant needed for each sample, and so the resulting turbidity is slightly different (though it is only observable via the UV/Vis spectrometer, not in photographs or to the naked eye). What is important here is that it is clear that there is effectively no change in particle size in any of the amidine latex/silica suspensions, confirming the stabilizing behavior of the nanoparticles that was observed visually. 


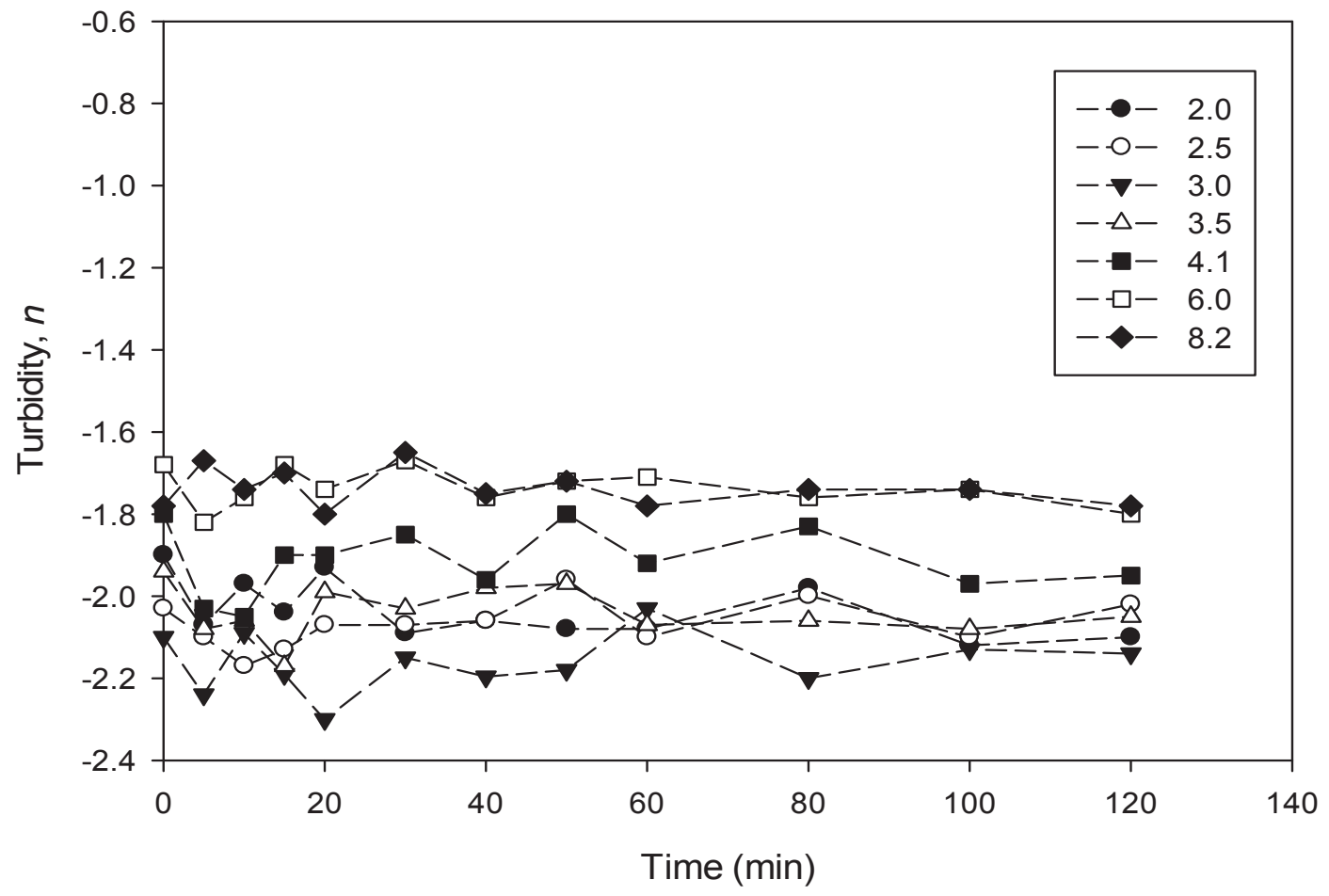

Figure 11: Flocculation behavior of $0.1 \% \mathrm{v}$ silica microspheres in solution with $0.5 \% \mathrm{v}$ amidine latex nanoparticles. The dotted lines serve only to guide the eye and connect the data points for a given sample. The $\mathrm{pH}$ values used are labeled in the legend.

\subsubsection{Adsorption of nanoparticles on silica surfaces}

The primary method of evaluating the interactions between the latex nanoparticles and the silica surfaces was through SEM imaging of silica that had been immersed in a nanoparticle solution. After adsorption and drying, the samples were sputter-coated with a 1-2 nm layer of gold before being imaged to produce a conductive surface and prevent charge accumulation. Figures $12 \mathrm{a}$ and $12 \mathrm{~b}$ show images of fused silica slides that had been immersed for 30 minutes in $0.1 \% \mathrm{v}$ sulfate latex solutions, at varying $\mathrm{pH}$ values. The level of adsorption observed is 
negligible for higher $\mathrm{pH}$ values, and becomes noticeable by $\mathrm{pH} 3.5$. Lower $\mathrm{pH}$ samples show significant adsorption, as the silica becomes positively charged.
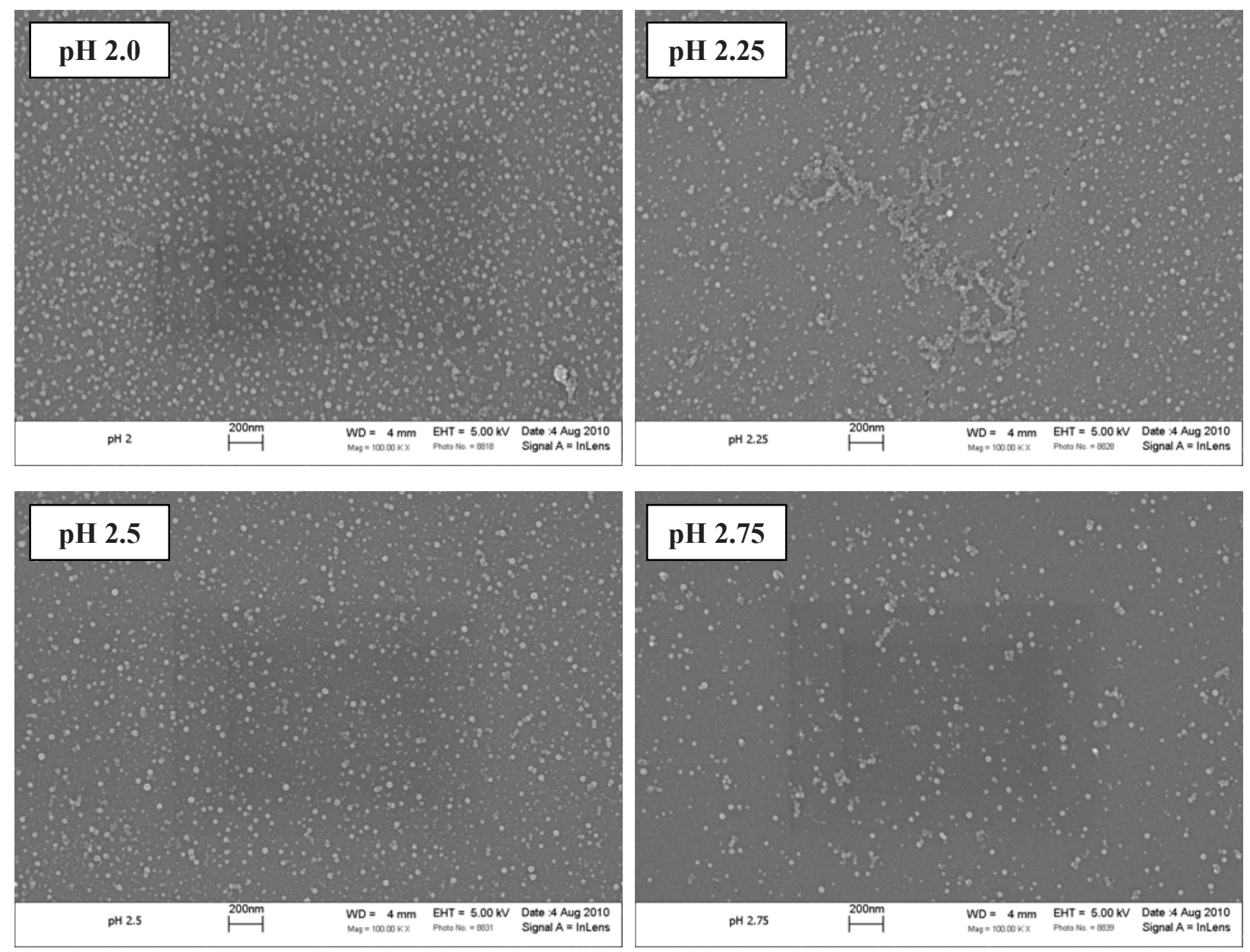

Figure 12a: SEM images of fused silica after immersion for $30 \mathrm{~min}$. in $0.1 \% \mathrm{v}$ sulfate latex, below $\mathrm{pH}$ 3.0. Images are $100 \mathrm{kX}$ magnification, showing increasing adsorption with decreasing $\mathrm{pH}$. 

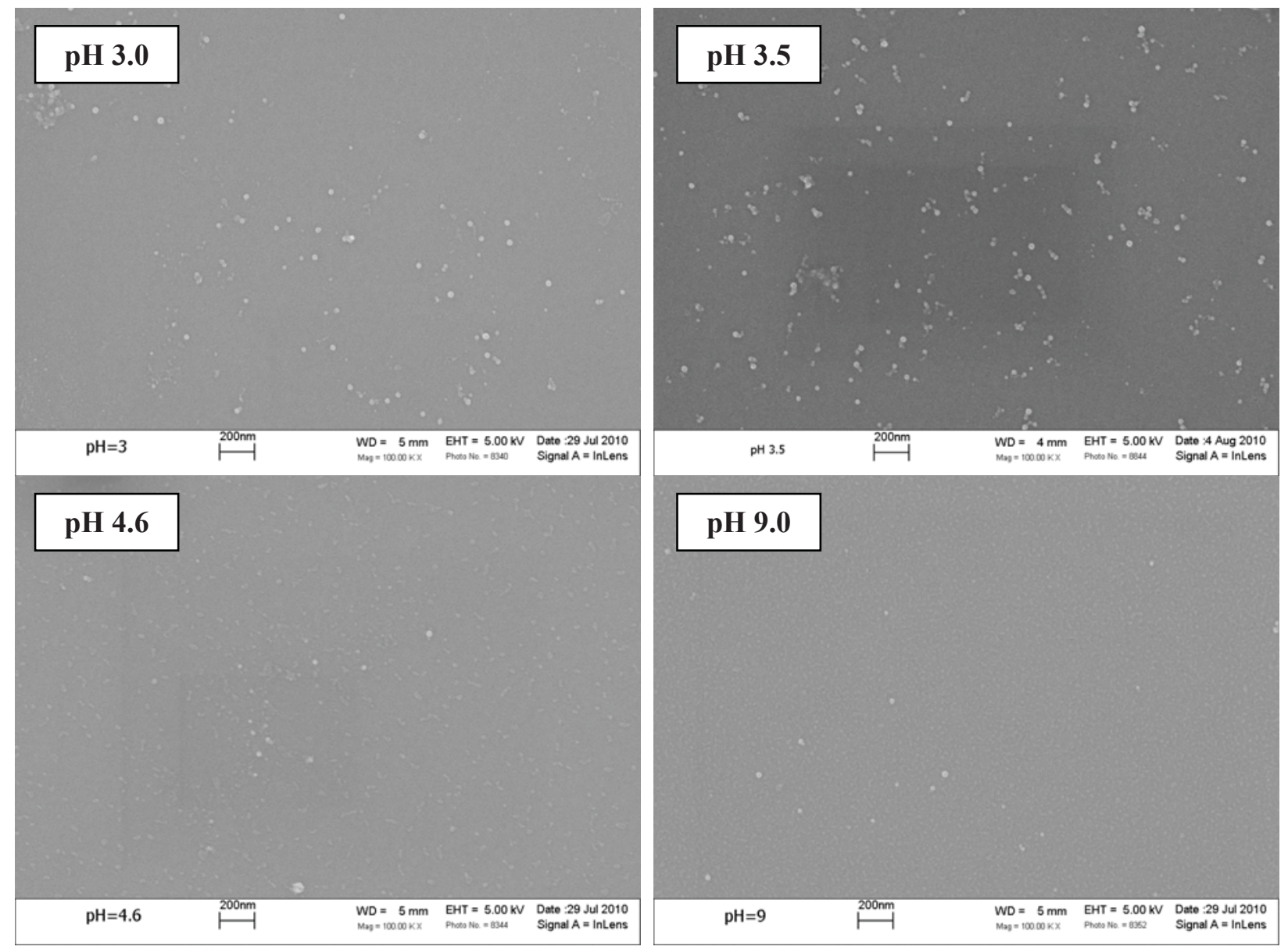

Figure 12b: SEM images of fused silica after immersion for $30 \mathrm{~min}$. in $0.1 \% \mathrm{v}$ sulfate latex, at $\mathrm{pH}$ values above 3.0. Images are $100 \mathrm{kX}$ magnification, showing increasing adsorption with decreasing $\mathrm{pH}$.

Estimates of the surface density of adsorbed particles were determined from the SEM images. Multiple images were taken at each $\mathrm{pH}$ and the average density was estimated. Figure 13 shows the approxmate number of nanoparticles per square micron at each to $\mathrm{pH}$. Above a $\mathrm{pH}$ of 4.5 there are effectively no adsorbed nanoparticles. By pH 3.5 there is noticable adsorption, which increases with decreasing $\mathrm{pH}$. 


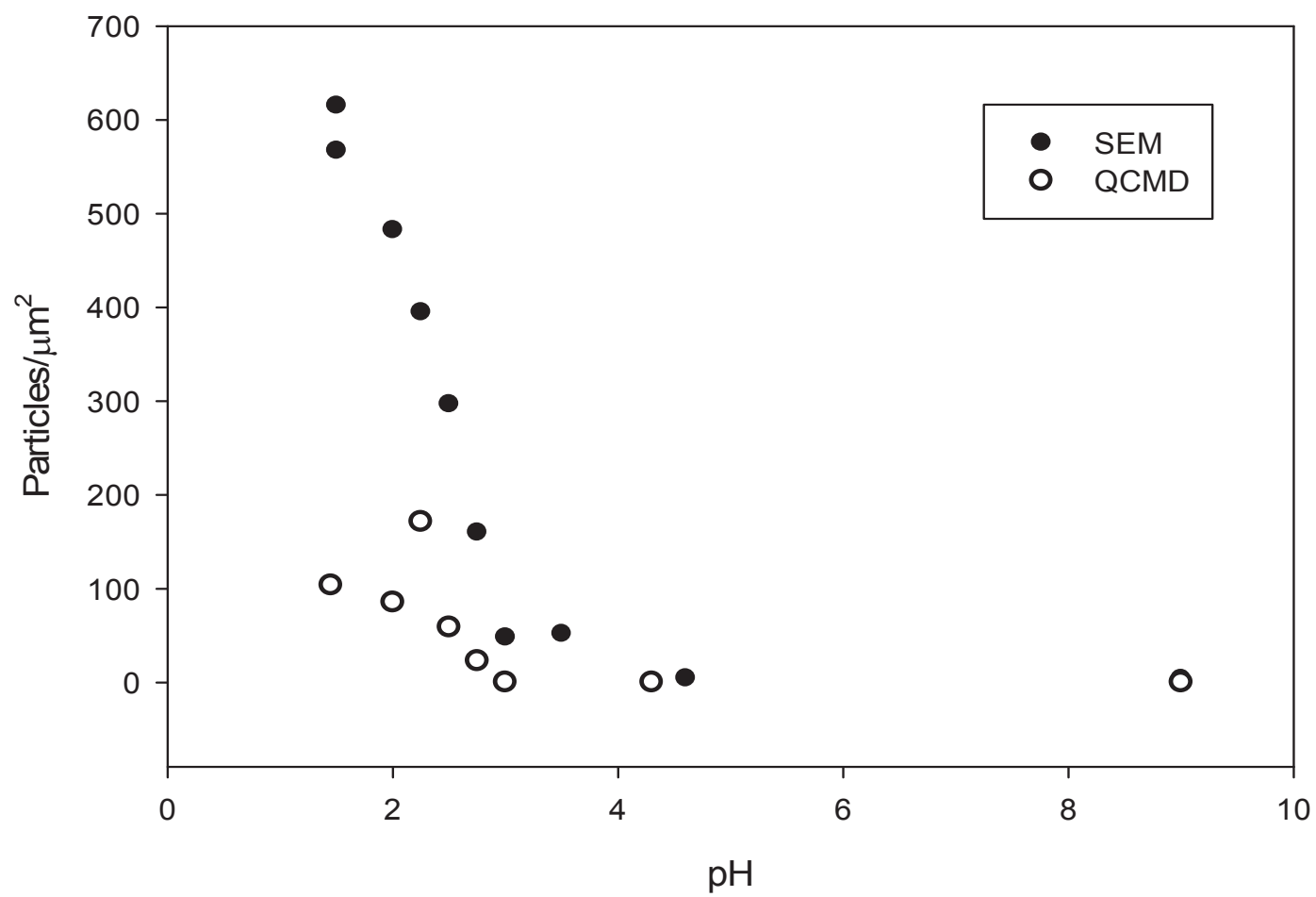

Figure 13: Surface density of adsorbed sulfate latex nanoparticles.

To confirm that adsorption was in fact taking place, rather than only non-adsorbed particles simply drying on the surface, QCMD measurements were done using the same concentration of sulfate latex particles, $0.1 \% \mathrm{v}$. The mass adsorbed was calculated using the Sauerbrey equation (Equation 7)[38], where $\Delta f$ is the change in crystal frequency, $f_{o}$ is the resonant frequency, $A$ is the active crystal area, $\rho$ is the density of quartz, $\mu$ is the shear modulus of quartz, and $\Delta m$ is the change in mass.

$$
\Delta f=-\frac{2 f_{o}}{A \sqrt{\rho \mu}} \Delta m
$$

The particle density was calculated from the adsorbed mass using the mean particle size $(22 \mathrm{~nm})$ and the density of the polystyrene particles. The measured particle density from the 
QCMD measurements is shown in comparison to the SEM estimates in Figure 13. The overall trend is similar, showing no adsorption above about $\mathrm{pH} 3.0$ with increasing adsorption below that point. The overall magnitude is not as large as the estimates from the SEM images.

It is important to stress that the densities from the SEM images are estimates, and that the difference in adsorbed density determined by the different method seen in Figure 13 could result from fundamental differences in the nature of the measurement. Specifically, the QCMD measurements are taken in a flow cell, with the nanoparticle solution slowly flowing over the surface, rather than simply a stationary immersion of the surface. Additionally, the surface is attached to a vibrating quartz crystal, which could account for differences between the two methods. Regardless, it is clear that adsorption of the nanoparticles on the surface is occuring.

The immersion adsorption experiments were done using the silica microspheres as well. The spheres shown in the top left image of Figure 14 (shown at $\mathrm{pH} 2.0$ ) were fixed to a silica substrate and immersed identically to the bare silica slides. These show that the nanoparticles adsorb to the spheres in the same way as the slides. 

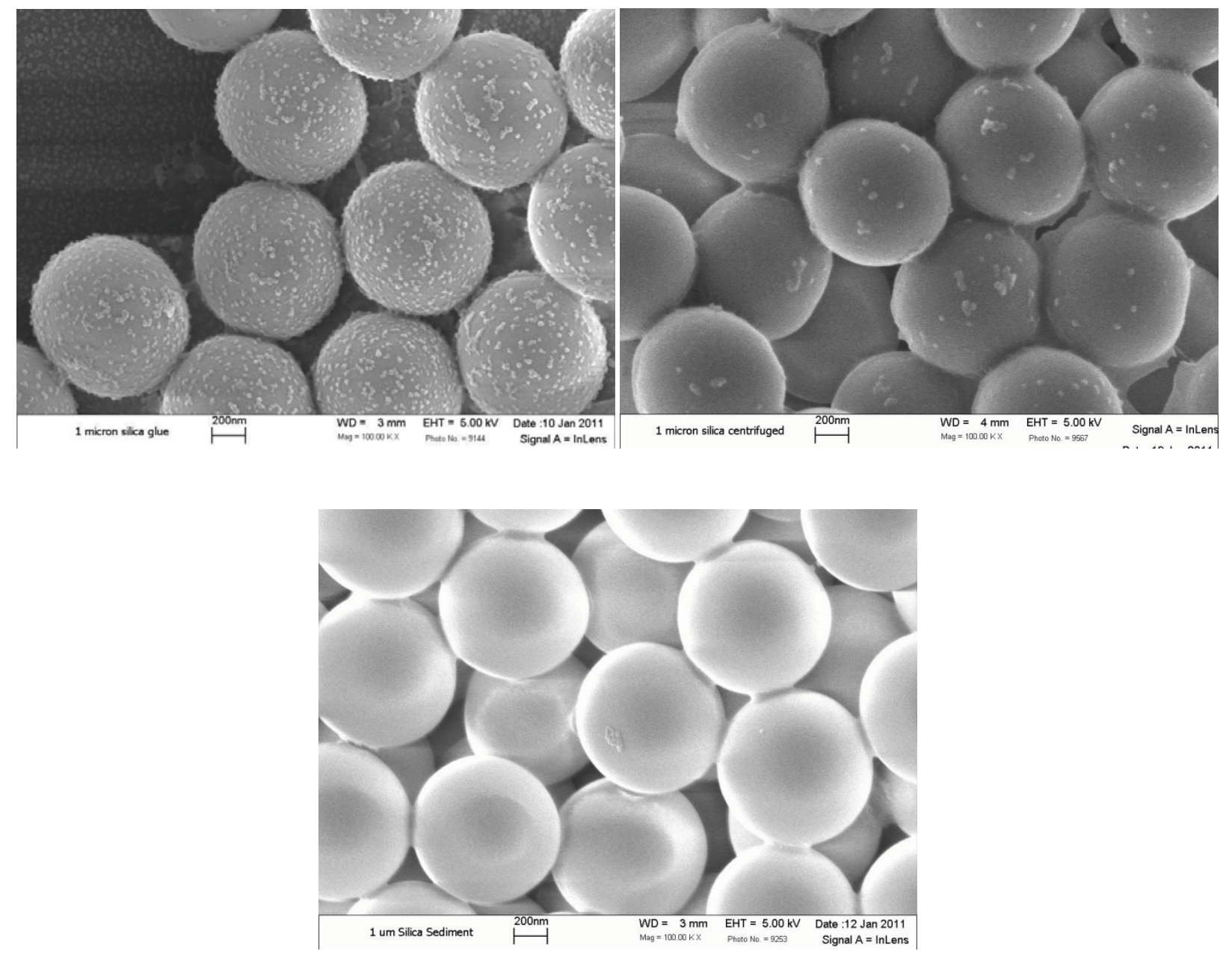

Figure 14: Adsorption of sulfate latex particles on the $1 \mu \mathrm{m}$ silica spheres, with different amounts of time spent in the rinse solution (at $\mathrm{pH} 2.0$ ). Top left: spheres were rinsed for a few seconds. Top right: spheres were rinsed (and centrifuged) for 5 minutes. Bottom: spheres were allowed to settle out of rinse solution over a couple hours.

Additionally, free spheres in solution were examined. Initially the spheres were allowed to sediment from the nanoparticle solution, and then the supernatant drawn off the sediment and replaced with a particle free rinse of water (titrated to the same $\mathrm{pH}$ ). In doing so, it was observed that the particles undergo desorption into the rinse solution if exposed for long enough time. The 
top right image of Figure 14 shows spheres that were rinsed for about 5 minutes (with sedimentation accelerated using a centrifuge). The bottom image shows spheres that sedimented by gravity alone and therefore were in the rinse for a couple of hours. The centrifuged sample shows more particles remaining than the longer sedimentation sample.

The effect of nanoparticle concentration on adsorption was examined in the range of $0.1 \% \mathrm{v}$ to $3.0 \% \mathrm{v}$ sulfate latex nanoparticles. Figure 15 shows the observed dependence of adsorption on the nanoparticle concentration. Between $0.1 \% \mathrm{v}$ and $0.5 \% \mathrm{v}$ there does not appear to be a particularly significant difference in the amount of adsorption. The higher concentrations $(1.0 \% \mathrm{v}$ and $3.0 \% \mathrm{v})$ start to show more adsorption, particularly in the form of regions containing particles that are more than 1 particle layer thick. 


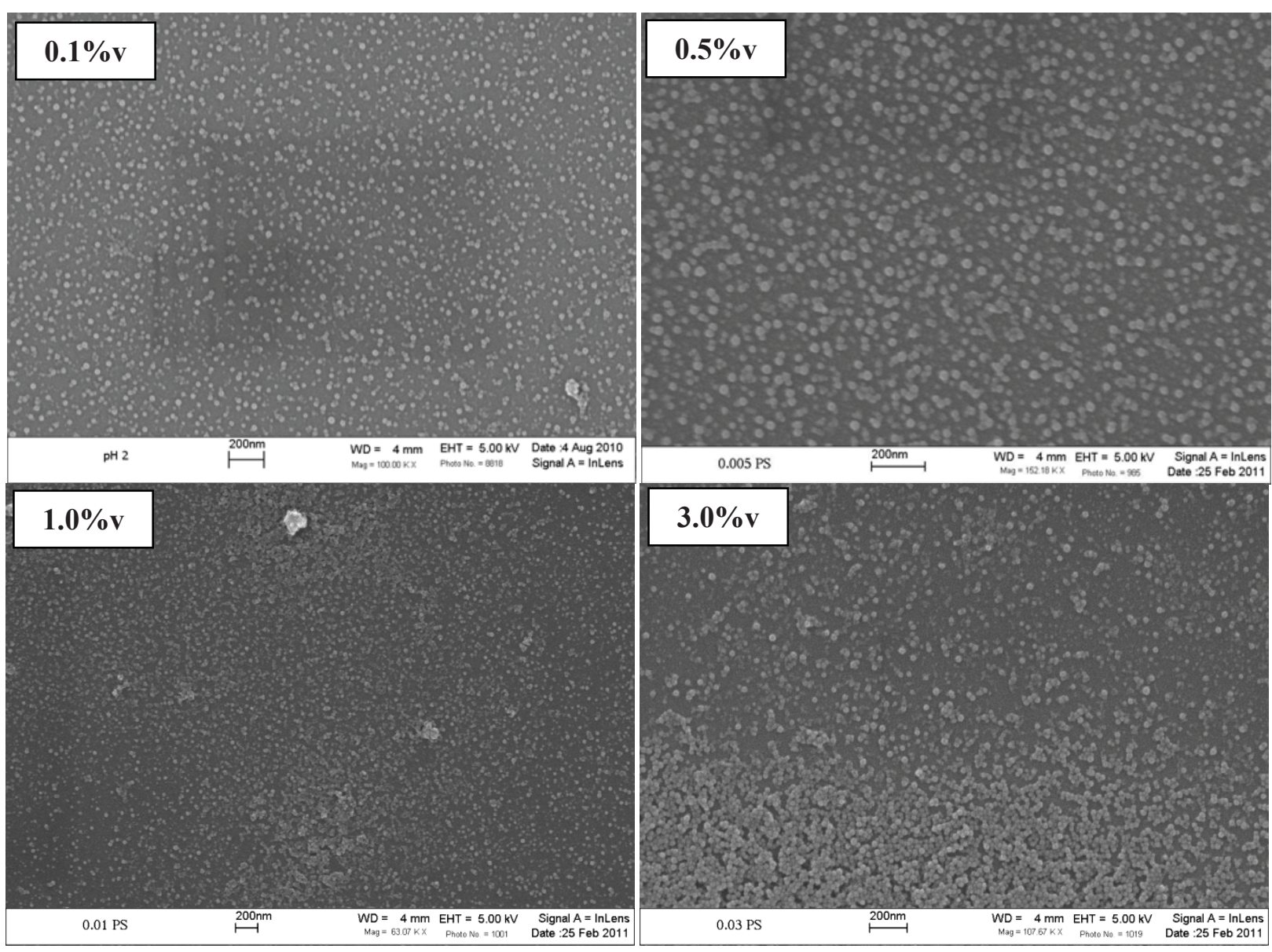

Figure 15: Sulfate latex nanoparticle adsorption on silica at $\mathrm{pH}$ 2.0, varying concentration of nanoparticles. Top left: $0.1 \% \mathrm{v}$. Top right: $0.5 \% \mathrm{v}$. Bottom left: $1.0 \% \mathrm{v}$. Bottom right: $3.0 \% \mathrm{v}$.

The $3.0 \% \mathrm{v}$ sample in particular contained regions of very high density of particles (though they were present at $1.0 \% \mathrm{v}$ and to a much lesser extent at lower concentrations), and Figure 16 compares two regions on the same surface. There are regions where the nanoparticles cover the surface to the point of being impossible to determine the thickness of the layer, and also regions where the silica substrate is clearly visible. This is most likely a result of the drying process. As the liquid interface recedes across a surface with more than one layer of nanoparticles, the upper layer or layers could be effectively pulled and concentrated in other locations, resulting in patches of different amounts of observed nanoparticles. 


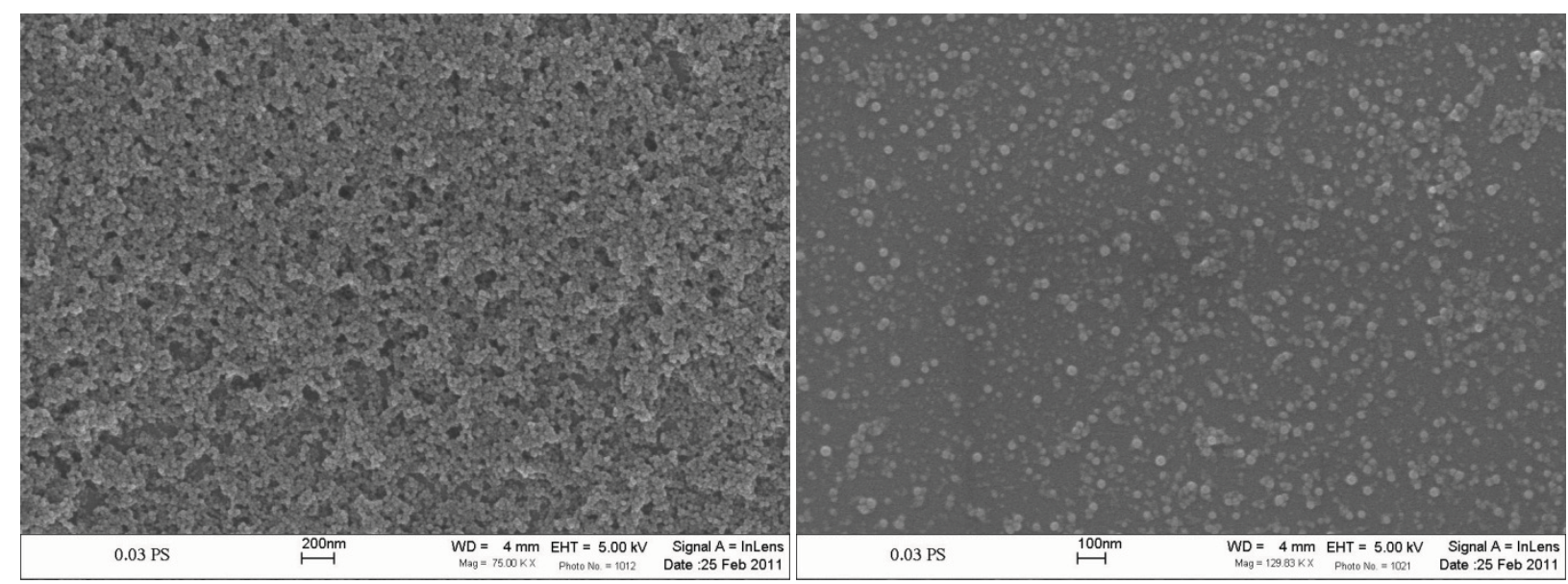

Figure 16: An example of contrasting regions of particle adsorption for $3.0 \% \mathrm{v}$ sulfate latex at pH 2.0.

Adsorption measurements using amidine latex nanoparticles were carried out identically to the experiments with the sulfate nanoparticles. The images in Figure 17 show the adsorption of the nanoparticles on silica slides at four $\mathrm{pH}$ values. In each case there is significant adsorption, far more than which was present using sulfate nanoparticles at the same conditions. The particle density on the surfaces actually appears to decrease with increasing $\mathrm{pH}$, which is essentially the opposite of what was expected, as the silica surface becomes more negatively charged with increasing $\mathrm{pH}$ while the zeta potential of the amidine latex nanoparticles remains highly positive over this $\mathrm{pH}$ range. Nonetheless, it is clear from the photographs that the adsorbed nanoparticle density is highest when both the silica plate and amidine latex nanoparticles are positively charged $(\mathrm{pH} 2.0)$. 

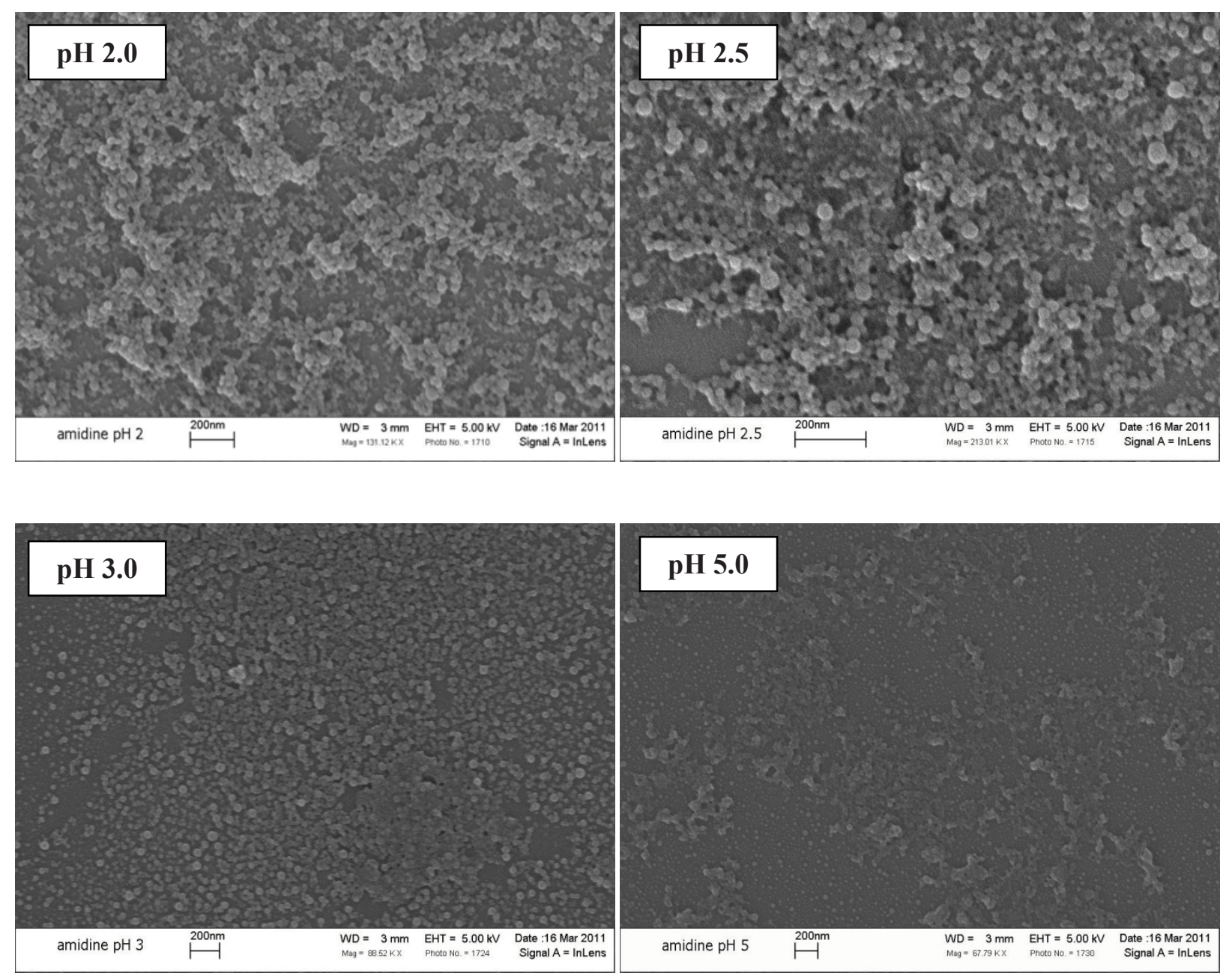

Figure 17: Silica surfaces with adsorbed amidine latex $(0.5 \% \mathrm{v}$ solution $)$ at varying $\mathrm{pH}$ values.

A comparison of images between sulfate latex and amidine latex is shown in Figure 18. Both images were done with $0.5 \% \mathrm{v}$ nanoparticles at $\mathrm{pH} 2.0$. The amidine particles clearly have adsorbed in much larger quantities than the sulfate particles. Since the amidine are present in such thicknesses, it is quite difficult to get an accurate quantitative estimate of the degree of difference in adsorption using these SEM images, but estimates of the thickness were made to determine approximate area densities of the particles. 

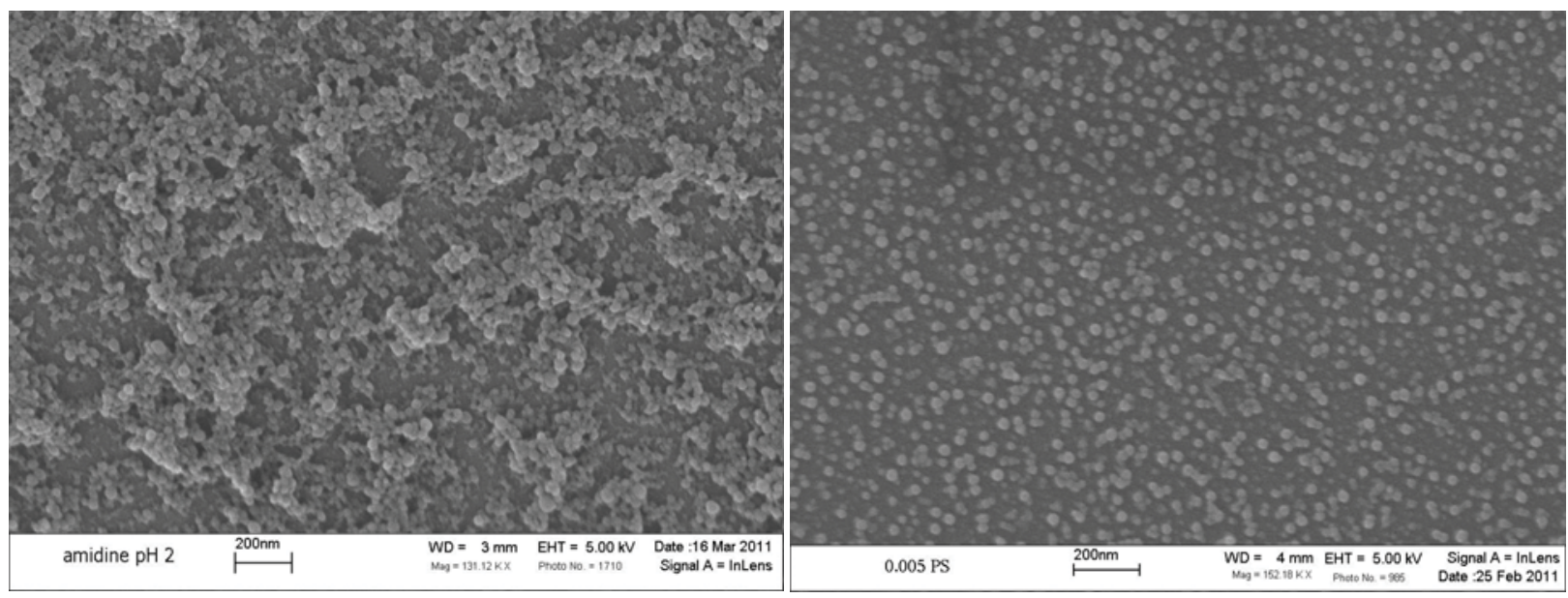

Figure 18: Comparison of amidine (left) and sulfate (right) latex adsorption at $\mathrm{pH} 2.0$ on silica slides. Nanoparticle solution concentration was $0.5 \% \mathrm{v}$.

The approximate observed particle densities for both amidine and sulfate latex particles are shown in Figure 19 for various $\mathrm{pH}$ values and nanoparticle concentrations of $0.5 \% \mathrm{v}$. The sulfate latex show slightly more adsorption at this concentration than at $0.1 \% \mathrm{v}$ (Figure 13), specifically at $\mathrm{pH} 5.0$. With $0.1 \% \mathrm{v}$ sulfate latex there was negligible adsorption above $\mathrm{pH} 4.5$, while there were over 200 particles/ $\mu \mathrm{m}$ observed while using $0.5 \% \mathrm{v}$. There was far less of a difference between concentrations at lower $\mathrm{pH}$ values. Regardless, the amidine nanoparticles adsorb in far higher quantities at every observed $\mathrm{pH}$, with at least three times more than the sulfate latex nanoparticles observed. This is a key difference between the two systems, which can potentially explain the difference in stability of binary solutions using the different particles. 


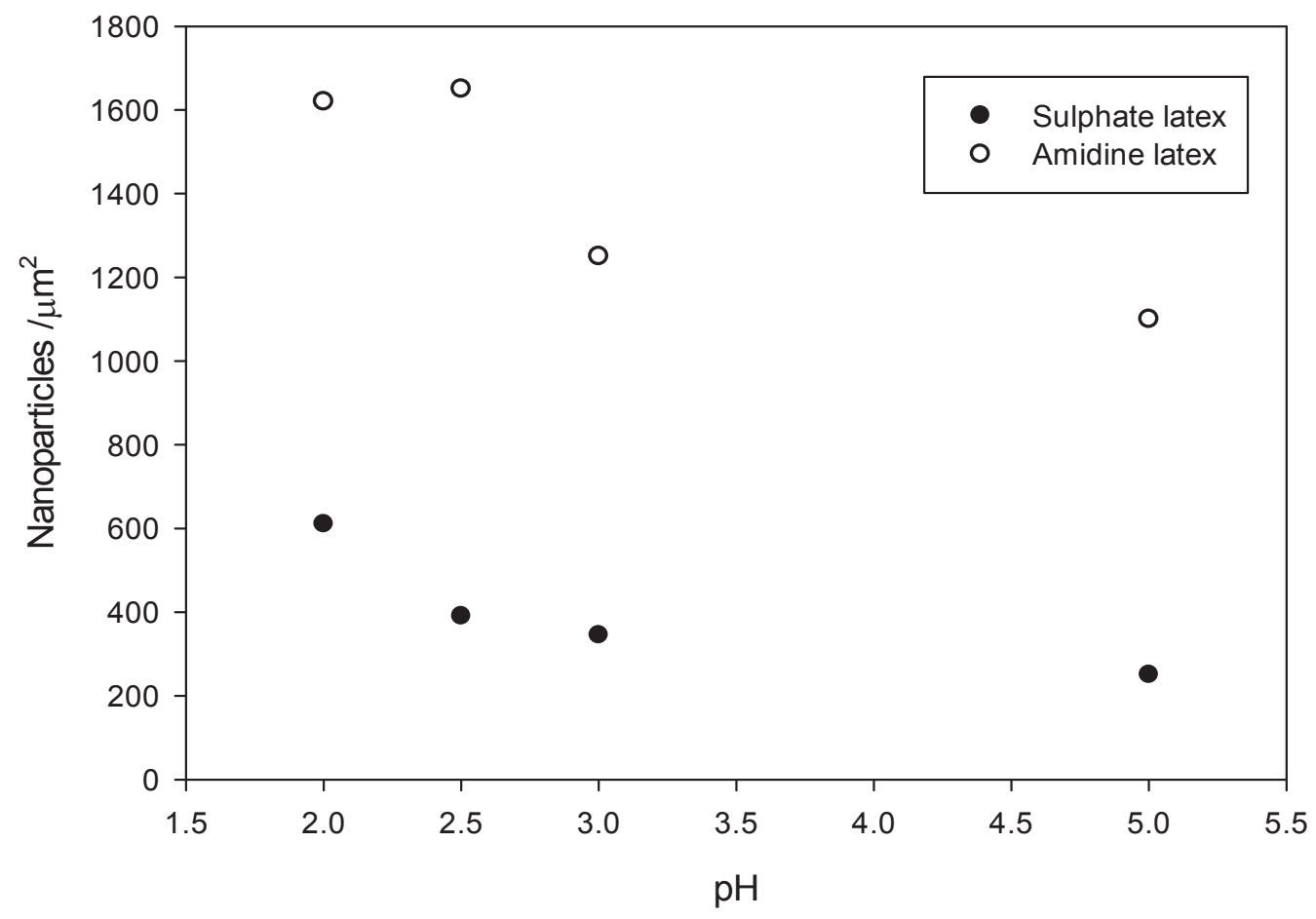

Figure 19: Comparison of approximate amidine latex and sulfate latex particle densities (both from $0.5 \% \mathrm{v}$ nanoparticle solutions) on silica slides at varying $\mathrm{pH}$. 


\subsection{Discussion}

The purpose of these experiments was to investigate the potential stabilizing effects of adding highly charged nanoparticles to weakly charged microspheres, which would otherwise flocculate due to the lack of sufficient electrostatic repulsion between the particles. The rationale was that if the system would favor adsorption of the nanoparticles on the surface of the microspheres, the microspheres would effectively be "charged up" to the point where they had sufficient surface potential to prevent flocculation. This nanoparticle adsorption was indeed observed in the cases where the microspheres were weakly charged, however it did not necessarily stabilize the solutions where it was expected to.

The solutions containing sulfate latex nanoparticles and silica microspheres were the systems with which the majority of experiments used. In no cases were the nanoparticles able to completely stabilize the microspheres. As the zeta potential of the silica approached zero from being highly negatively charged, the degree of adsorption of the negatively charged latex particles increased as anticipated. There was increasing adsorption with increasing nanoparticle concentration as well. Looking at Figures 11, 12, 13, and 15, it is clear that the particles adsorbed in relatively large quantities, over 600 particles per $\mu \mathrm{m}^{2}$ as the $\mathrm{pH}$ approached 2.0 , or an approximate surface coverage of $24 \%$ (this is a rough estimate using the cross-sectional area of a $22 \mathrm{~nm}$ sphere as the area covered by a single particle; it should be noted that this estimation would not quite equal $100 \%$ coverage even with particles ideally packed on the surface as there would theoretically be gaps between the spherical particles).

From the zeta potential data and flocculation experiments, we see that the silica begins to flocculate in solutions below a $\mathrm{pH}$ of about 4.5 , solutions in which the microspheres had zeta potentials below about $-40 \mathrm{mV}$. Taking this value as an approximate threshold for what zeta 
potential would be necessary to maintain a stable suspension of silica, it would be expected that a more highly charged effective potential for the particles would provide stability. However, based on the zeta potential measurements on the binary solution of silica and sulfate latex (Table 1), the zeta potential does not appear to be the defining factor in stability.

The composite measurements all showed effective zeta potentials much larger than what was expected to be necessary to stabilize the particles, particularly at $\mathrm{pH} 3.0$ where the zeta potential of the silica in solution with sulfate latex particles was observed to be larger than -100 $\mathrm{mV}$. Comparatively, the silica alone had almost effectively zero potential at the same $\mathrm{pH}$ (and it flocculated rapidly). Yet, as seen in the flocculation photographs as well as the plots of the turbidity exponent, there was no quantifiable stabilization effect or even a slowing of the rate of flocculation.

The only possibility of the nanoparticles having any effect on the silica stability, in the observed cases, was at $\mathrm{pH}$ 2.0. However, even this was not consistently observed in these experiments. In Figure 8, it appears to be semi stable, however at other times (including Figure 7) it flocculated in the same way as the silica-only sample did at $\mathrm{pH}$ 2.0. Additionally, the composite zeta potential at $\mathrm{pH} 2.0$ was measured to be somewhat less negative than it was at $\mathrm{pH}$ 3.0. This seems somewhat backwards compared to expectations, as the samples which exhibited higher levels of nanoparticle adsorption had a lower composite zeta potential than one where there was less adsorption, since the change in potential from the silica-only solutions should arise solely from the nanoparticles. This could be explained by the slight difference in nanoparticle zeta potential between $\mathrm{pH} 3.0$ and 2.0. Even though the particles are supposed to have effectively constant zeta potentials over this $\mathrm{pH}$ range, based on Figure 3 they are slightly less 
negative (approximately $10 \mathrm{mV}$ ) at $\mathrm{pH} 2.0$, which could be enough to lower the composite zeta potential.

The amidine latex nanoparticles produced drastically different results for the binary solutions, most notably in that they prevented flocculation of the silica at every $\mathrm{pH}$ value that was observed. Here there is no doubt in the stability of the particles, as there was with the occasionally observed reduction in flocculation rate of the $\mathrm{pH} 2.0$ silica/sulfate. Once again, the addition of the nanoparticles results in a much larger composite zeta potential (around $+80 \mathrm{mV}$ ) than for the silica alone.

However, there is a very significant difference in the degree of adsorption of the amidine latex particles between the amidine and sulfate latex. The particle density estimates, shown in Figure 19 compared to the density of the sulfate latex particles, are at least triple the percent surface coverage that occurred using the sulfate latex. Most regions with more than one layer of nanoparticles appeared to be approximately three to four particle layers deep, and so the visible particles were counted and then multiplied by the apparent number of layers, which is not as qualitatively accurate as when it is possible to see all the particles. However it is quite clear even from casual observation that are multiple times more amidine latex particles at a given $\mathrm{pH}$ and concentration than there are sulfate particles at the same conditions. Based on these density estimates, the percent coverage of about $70-75 \%$ compared to about $24 \%$ for the sulfate latex particles (again using the rough estimation of surface area covered by a single particle).

It is this increased level of adsorption that appears responsible for the stabilizing effect of the amidine latex. While the sulfate latex particles exhibit extensive and typically uniform coverage of the silica surfaces at low $\mathrm{pH}$ values, at none of the $\mathrm{pH}$ values do the particles 
completely cover the surface, as shown in Figures 11,16 , and 17 . At $0.5 \%$ v sulfate latex and lower there remain gaps between the particles, and the underlying silica substrate is still visible Lewis and coworkers found that nanoparticle concentrations above about $1 \% \mathrm{v}$ caused flocculation due to depletion attractions [27, 28], so even if sufficient coverage was achieved at the higher concentrations, it is likely that flocculation would still occur.

The presence of exposed silica indicates the likelihood that the surface potential is locally variable, which would not be indicated in a measurement of the composite zeta potential of silica and nanoparticle solutions as measured by microelectrophoresis. This method of determining the zeta potential uses the translational velocity of the particles in an electric field to calculate the electrophoretic mobility. As such, it can only produce the average zeta potential over the particle surface, with no consideration of surface inhomogeneity [39, 40]. The silica microspheres used in these experiments are sufficiently Brownian that all possible mutual orientations would be sampled during the interaction between two microparticles. As such, any patches available for favorable aggregation on one particle would eventually align with another patch on a second particle. Therefore, the weakly or negligibly charged regions on two silica microspheres may be able to still come into contact favorably.

This heterogeneous distribution of surface charge due to the adsorption of latex nanoparticles would be a likely explanation for the continued flocculation of the silica particles even when the effective zeta potential is more highly charged than what was necessary to maintain stability in silica-only solutions. In systems where the average surface or zeta potential over the whole surface of a colloidal particle would indicate a sufficiently repulsive force to prevent aggregation or deposition, it has been shown that attraction and deposition still occurs [41-44]. This is typically attributed to heterogeneities in the surface charge, with surface patches 
that exist where attractive forces dominate over the surface-average repulsion, leading to particle deposition.

In particular, the work of Santore and coworkers has shown that even comparatively small densities of heterogeneous surface patches can lead to large degrees of particle adsorption on surfaces [41, 42]. In these experiments, cationic patches $10 \mathrm{~nm}$ in size were placed on negatively charged silica substrates, producing a surface that had a negative charge averaged over the entire surface, but contained randomly distributed attractive patches. A solution containing negatively charged silica microspheres was flowed over the surface. The results of measurements of particle attraction and adhesion show that upon reaching a critical density of positive patches, though well below the $50 \%$ coverage that would result in a net zero charge surface, the silica spheres would adhere to the surface. In solutions with longer Debye lengths (low ionic strength), the critical patch percentage was observed around $10 \%$, or an average spacing of about $34 \mathrm{~nm}$ between patches, at which the maximum attainable adhesion was quickly reached. Higher ionic strengths resulted in measureable adhesion at lower densities [41, 42].

In Figure 15, the sulfate latex nanoparticles are adsorbed to the surface of the silica in generally small groups of only a few particles at all the observed concentrations. The gaps in between the particles are on the order of the particle size or larger. Based on an average particle size of $22 \mathrm{~nm}$, the exposed patches of silica are larger and more numerous than those described by Santore, being more than twice the size and spaced more closely (assuming, based on the SEM images, an approximate size and spacing of the patches to almost identical to the nanoparticle size of $22 \mathrm{~nm}$ ). And while the two systems are not identical, the prevalence of patches of exposed weakly-charged silica (in this case due to van der Waals attractions rather 
than adsorbed polycations) means it is highly likely that the observed flocculation of the silica microspheres is a direct consequence of the surface patchiness.

The amidine particles exhibit superior coverage of the surfaces, both in thickness and the amount of exposed space between adsorbed particles. A more complete coverage by the nanoparticles would sufficiently eliminate any patchiness that would lead to flocculation of the silica microspheres. As visible in Figure 18, the comparison of amidine latex and sulfate latex patchiness reveals that the exposed patches of silica with the adsorbed amidine latex nanoparticles are much fewer and farther in between than for the sulfate latex particles.

Taking the degree of adsorption as the main condition for the nanoparticles to stabilize the silica, the focus shifts to why the amidine particles would be able to adsorb in greater quantities. Beyond just the amount of adsorbed particles, the amidine particles behave somewhat differently than the sulfate particles in that the degree of adsorption does not seem to correlate with the zeta potential of the silica. The amidine particles are positively charged and for the bulk of the observed $\mathrm{pH}$ range, they are oppositely charged with regards to the silica. However, rather than exhibiting increasing adsorption with decreasing electrostatic repulsion, the adsorption appears to be somewhat independent or even the opposite behavior. The degree of adsorption is higher at $\mathrm{pH} 2.0$ and 2.5, where both the silica and amidine latex are positively charged (albeit the silica is only weakly charged). At $\mathrm{pH} 5.0$, where the silica is significantly negatively charged, and where it would be expected to be more strongly attracted to the surface, the density of adsorbed particles at this $\mathrm{pH}$ was the least of all observed $\mathrm{pH}$ values.

As a rough approximation, the patch size necessary for two sphere surfaces to approach within a Debye length of separation $(x)$ was calculated using the simple geometry of a $1 \mu \mathrm{m}$ 
sphere with $22 \mathrm{~nm}$ nanoparticles adsorbed on the surface. Figure 20 shows a simple schematic of two approaching silica sphere surfaces with adsorbed nanoparticles. The geometry shown is representative of the maximum required patch size for a surface, where the adsorbed nanoparticles are symmetrical between the two spheres, thus requiring the patch be large enough that the separation $(x)$ is uniform between the different particles. Using this configuration, the patch size needs to be approximately $275 \mathrm{~nm}$ across to allow the surfaces to approach close enough to allow the silica to flocculate.

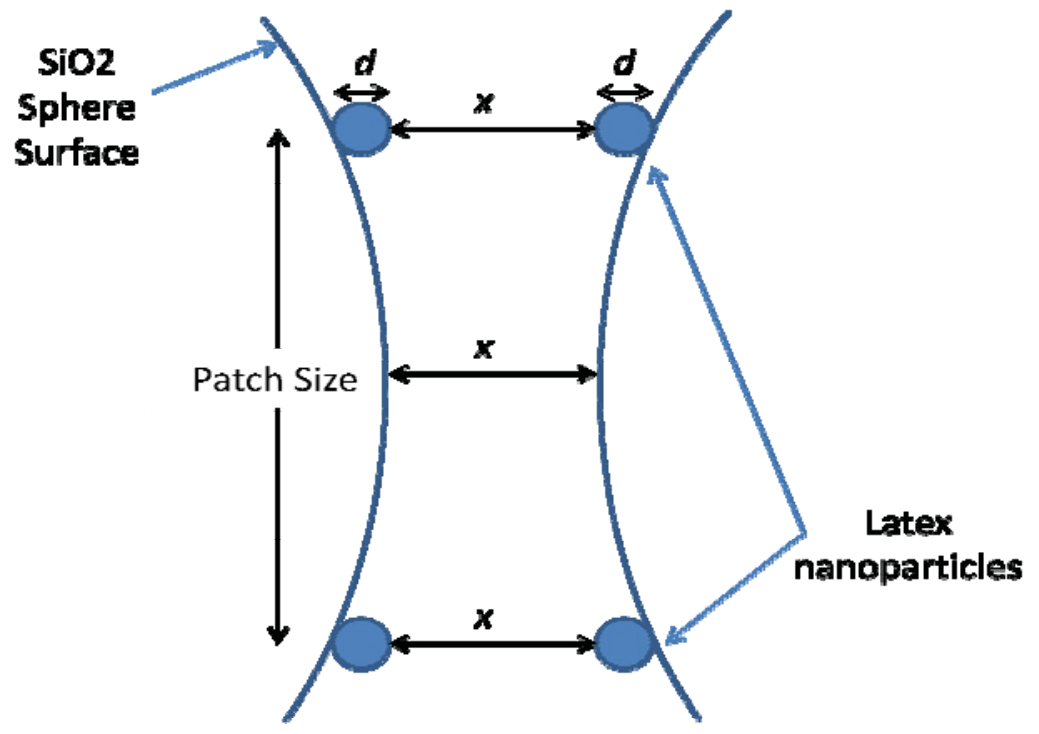

Figure 20: Schematic of the maximum patch size needed for two silica sphere surfaces to come into contact. The variable $x$ is the separation distance; $d$ is the nanoparticle diameter.

Based on the SEM images of the sulfate latex on the silica surfaces (Figures 12, 14, 15, 16) the particles are typically evenly distributed, however, there are patches in the $100-150 \mathrm{~nm}$ range visible. Additionally, these were the most uniform cases, and there were areas on surfaces (not shown in this thesis due to being less representative of the majority of observed surfaces) with larger patches. The minimum patch size calculated in the geometry of Figure 20, however, 
is just a rough approximation of what is effectively the worst-case scenario. If the nanoparticles were configured so that they did not come into direct contact with the particles adsorbed on the second sphere, this would result in a smaller required patch size. Therefore, even though estimated patch size is somewhat larger than what is typically observed in the surface images, it is reasonable to conclude that the observed patches are sufficiently sized to allow for flocculation.

It would seem that the particles would be identical in every aspect except for surface charge, as they are manufactured to the same size specification $(0.02 \mu \mathrm{m})$ by the same manufacturer, but the particles differ in a couple potentially significant ways, as indicated by the certificates of analysis provided by the distributor. Most notably, the amidine latex particles have a greater surface charge density than the sulfate latex, with $3.0 \mu \mathrm{C} / \mathrm{cm}^{2}$ for the amidine compared to $2.2 \mu \mathrm{C} / \mathrm{cm}^{2}$ for the sulfate latex, for $40 \%$ greater charge density. This is due to the area per amidine group being smaller than sulfate ( $528 \AA^{2}$ for amidine, $716 \AA^{2}$ for sulfate).

Additionally, the amidine latex particles are slightly more polydisperse than the sulfate latex, with a coefficient of variation (CV) of diameter of $21.9 \%$ compared to $14.7 \%$ for the sulfate latex particles. The slightly wider distribution means there would be somewhat more small particles present, which could allow for better surface coverage as the small particles would pack between larger ones. Figure 21 shows the Gaussian probability density function for the sulfate and amidine latex nanoparticles. 


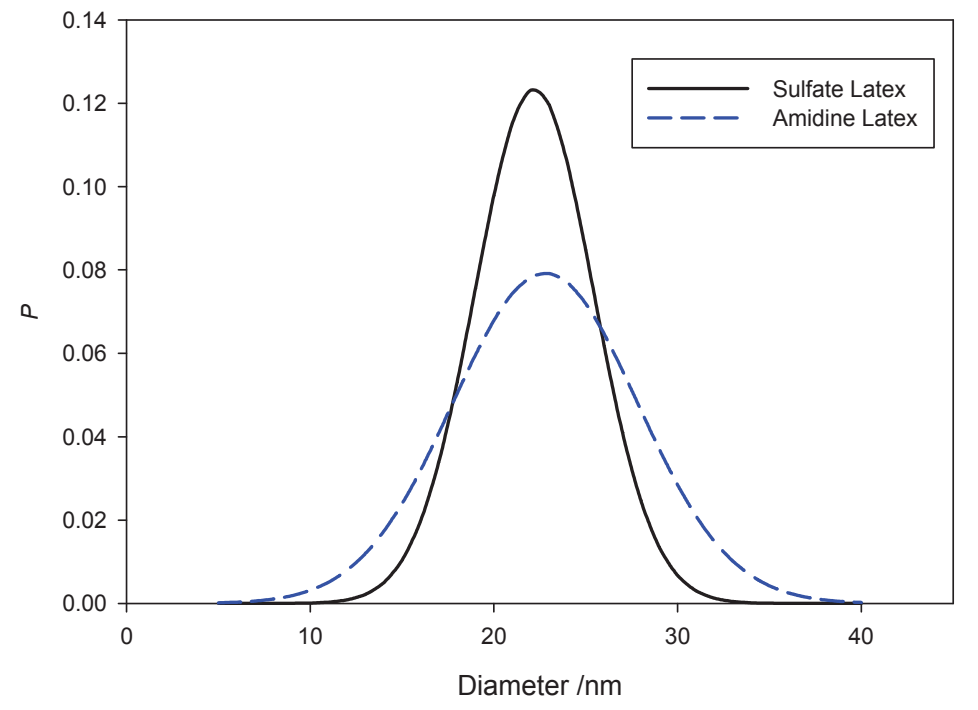

Figure 21: Probability density curves for the two types of nanoparticles used. Curves are based on data from the certificates of analysis provided from the supplier. Sulfate latex: The mean is $22 \mathrm{~nm}$ and $\mathrm{CV}$ is $14.7 \%$. Amidine Latex: the mean is $23 \mathrm{~nm}$ and $\mathrm{CV}$ is $21.9 \%$.

There are likely additional differences in surface chemistry between the nanoparticles, though without additional study and characterization of the particles and of the inter-particle forces, it would be difficult to speculate exactly what they might be. However, during the course of these experiments, there were some difficulties in using the amidine latex particles that were not present with the sulfate latex particles. Specifically, attempts to accurately size the amidine particles were somewhat unsuccessful when using both the Zetasizer and TEM imaging. In short, the Zetasizer, which operates via dynamic light scattering, had difficulty obtaining clean data, and the particles TEM images were difficult to distinguish (almost no contrast in between particles and the background), while there were no difficulties when using the sulfate latex particles (see Appendix B for more information). Ultimately, these may not be significant, but it 
is worth noting these differences since the amidine and sulfate latex particle behavior differed significantly in their interactions with the silica.

To completely understand these systems, future work on the particle interactions is necessary, as well as further characterization of the differences between the nanoparticles. It will be important to better understand the role of surface coverage of nanoparticles on the microspheres has in the effectiveness of the stability of the particles. Additional investigation of the limits of the parameters such as nanoparticle or microsphere concentration, ionic strength, and $\mathrm{pH}$ is necessary to determine what range conditions that the microspheres can be stabilized. 


\section{Chapter 4}

\section{Conclusions}

These experiments were done in order to explore the possibility of stabilizing colloidal solutions of weakly charged microspheres (which at low $\mathrm{pH}$ values lack significant electrostatic repulsion, allowing for rapid flocculation) using highly charged nanoparticles. From the results, the following conclusions can be drawn:

1. At low $\mathrm{pH}$ values, the $1 \mu \mathrm{m}$ silica spheres are very weakly charged $(<10 \mathrm{mV}$ between $\mathrm{pH}$ 2.0 and 3.0). The addition of either negatively charged sulfate latex or positively charged amidine latex nanoparticles causes the effective zeta potential to increase dramatically in this $\mathrm{pH}$ range $(-63.2 \mathrm{mV}$ to $-108.1 \mathrm{mV}$ and $+79.6 \mathrm{mV}$ to $+83.1 \mathrm{mV}$ for sulfate and amidine latex, respectively) as measured using microelectrophoresis, which utilized translational particle mobility to calculate an average potential over the surface.

2. In the cases with weakly charged silica, the addition of latex nanoparticles results in adsorption of the particles onto the silica sphere surfaces. The degree of adsorption of the sulfate latex particles is significantly less than that of the amidine latex particles. The maximum observed adsorption for both was at $\mathrm{pH} 2.0$ with around 600 sulfate latex particles per $\mu \mathrm{m}^{2}$ (approximately $24 \%$ coverage) and over 1600 amidine latex particles per $\mu \mathrm{m}^{2}$ (approximately $70 \%$ coverage). For the amidine latex particles, this is much more of an estimate do to the indeterminate number of layers of particles in many adsorbed regions of the surface. Consequently, there is much more exposed silica substrate when using sulfate latex particles compared to using amidine latex nanoparticles. 
3. The silica alone flocculates at low $\mathrm{pH}$ values in a matter of minutes, sedimenting almost completely in 30 minutes. With the addition of the positively charged amidine latex nanoparticles, there is no observed flocculation at any $\mathrm{pH}$ value and the microspheres sediment as individual particles, taking hours to sediment completely. However, the sulfate latex was never observed to reliably stabilize the microspheres, at any $\mathrm{pH}$ or concentration.

4. Based on the measured zeta potentials of silica with adsorbed polystyrene nanoparticles, it can be concluded that nano-scale heterogeneity in the form of exposed silica patches between nanoparticles are sufficient to allow for the silica microspheres to flocculate. Consequently, the net or average surface charge of the microspheres is not a sufficient indicator of the particle stability, as the silica continued to flocculate even though the measured zeta potential of silica with adsorbed sulfate latex particles significantly exceeded that which was necessary to stabilize the silica-only suspensions. As the amidine particles provided superior coverage of the silica surface, there were very few exposed patches, and the microspheres remained stable. 


\section{$\underline{\text { References }}$}

1. Liu, J. and E. Luijten, Stabilization of Colloidal Suspensions by Means of Highly Charged Nanoparticles. Phys Rev Lett, 2004. 93(24).

2. Ringenbach, E., G. Chauveteau, and E. Pefferkorn, Effect of soluble aluminum ions on polyelectrolyte-alumina interaction. Kinetics of polymer adsorption and colloid stabilization. Colloid Surface A, 1995. 99: p. 161-173.

3. Uskokovic, V., R. Odsinada, S. Djordjevic, and S. Habelitz, Dynamic light scattering and zeta potential of colloidal mixtures of amelogenin and hydroxyapatite in calcium and phosphate rich ionic milieus. Arch Oral Biol, 2011. 56(6): p. 521-32.

4. Mosley, L.M., K.A. Hunter, and W.A. Ducker, Forces Between Colloid Particles in Natural Waters. Environ Sci Technol, 2003. 37(15): p. 3303-3308.

5. Karathanasis, A. and D. Johnson, Stability and transportability of biosolid colloids through undisturbed soil monoliths. Geoderma, 2006. 130(3-4): p. 334-345.

6. Hiemenz, P.C. and R. Rajagopalan, Principles of Colloid and Surface Chemistry. 3rd ed1997: CRC Press.

7. Karanikas, S. and A. Louis, Dynamic Colloidal Stabilization by Nanoparticle Halos. Phys Rev Lett, 2004. 93(24).

8. Puls, R.W., C.J. Paul, and D.A. Clark, Surface chemical effects on colloid stability and transport though natural porous media. Colloid Surface A, 1993. 73: p. 287-300.

9. Deshiikan, S.R., E. Eschenazi, and K.D. Papadopoulos, Transport of colloids through porous beds in the presence of natural organic matter. Colloid Surface A, 1998. 145: p. 93-100.

10. Smay, J.E., G.M. Gratson, R.F. Shepherd, J. Cesarano III, and J.A. Lewis, Directed colloidal assembly of 3D periodic structures. Adv Mater, 2002. 14(18): p. 1279-1283.

11. Ash, S.G. and E.J. Clayfield, Effect of Polymers on the Stability of Colloids. J Colloid Interf Sci, 1976. 55(3): p. 645-657.

12. Sharma, A., S.N. Tan, and J.Y. Walz, Measurement of Colloidal Stability in Solutions of Simple, Nonadsorbing Polyelectrolytes. J Colloid Interf Sci, 1997. 190: p. 392-407.

13. Baird, J.C. and J.Y. Walz, The effects of added nanoparticles on aqueous kaolinite suspensions. I. Structural effects. J Colloid Interf Sci, 2006. 297(1): p. 161-9.

14. Fazelabdolabadi, B., J.Y. Walz, and P.R. Van Tassel, Influence of Charged Nanoparticles on Colloidal Forces: a Molecular Simulation Study. J Phys Chem B, 2009. 113: p. 13860-13865.

15. Rasa, M., A.P. Philipse, and J.D. Meeldijk, Heteroaggregation, repeptization and stability in mixtures of oppositely charged colloids. J Colloid Interf Sci, 2004. 278(1): p. 115-25.

16. Sharma, A., S.N. Tan, and J.Y. Walz, Effect of Nonadsorbing Polyelectrolytes on Colloidal Interactions in Aqueous Mixtures. J Colloid Interf Sci, 1997. 191: p. 236-246.

17. Snowden, M.J., S.M. Clegg, P.A. Williams, and I.D. Robb, Flocculation of silica particles by adsorbing and non-adsorbing polymers. J Chem Soc, 1991. 87(14): p. 2201.

18. Liu, Y., L. Gao, and J. Sun, Effect of acrylic copolymer adsorption on the colloidal stability of a 3Y-TZP suspension. J Eur Ceram Soc, 2002. 22: p. 863-871.

19. Liufu, S., H. Xiao, and Y. Li, Adsorption of poly(acrylic acid) onto the surface of titanium dioxide and the colloidal stability of aqueous suspension. J Colloid Interf Sci, 2005. 281(1): p. 155-63.

20. Liu, J. and E. Luijten, Colloidal stabilization via nanoparticle halo formation. Phys Rev E, 2005. 72(6).

21. Fleer, G.J. and J.M.H.M. Scheutjens, Block copolymer adsorption and stabilization of colloids. Colloid Surface, 1990. 51: p. 281-298. 
22. Zhulina, E.B., O.V. Borisov, and V.A. Priamitsyn, Theory of Steric Stabilization of Colloid Dispersions by Grafted Polymers. J Colloid Interf Sci, 1990. 137(2): p. 495-511.

23. Karimian, H. and A.A. Babaluo, Halos mechanism in stabilizing of colloidal suspensions: Nanoparticle weight fraction and pH effects. J Eur Ceram Soc, 2007. 27(1): p. 19-25.

24. Martinez, C.J., J. Liu, S.K. Rhodes, E. Luijten, E.R. Weeks, and J.A. Lewis, Interparticle interactions and direct imaging of colloidal phases assembled from microsphere-nanoparticle mixtures. Langmuir, 2005. 21(22): p. 9978-89.

25. Tohver, V., J.E. Smay, A. Braem, P.V. Braun, and J.A. Lewis, Nanoparticle halos: a new colloid stabilization mechanism. Proc Natl Acad Sci U S A, 2001. 98(16): p. 8950-4.

26. Zhang, F., G.G. Long, P.R. Jemian, J. Ilavsky, V.T. Milam, and J.A. Lewis, Quantitative Measurement of Nanoparticle Halo Formation around Colloidal Microspheres in Binary Mixtures. Langmuir, 2008. 24(13): p. 6504-6508.

27. Chan, A.T. and J.A. Lewis, Electrostatically Tuned Interactions in Silica MicrospherePolystyrene Nanoparticle Mixtures. Langmuir, 2005. 21: p. 8576-8579.

28. Chan, A.T. and J.A. Lewis, Size Ratio Effects on Interparticle Interactions and Phase Behavior of Microsphere-Nanoparticle Mixtures. Langmuir, 2008. 24(20): p. 11399-11405.

29. Gilchrist, J.F., A.T. Chan, E.R. Weeks, and J.A. Lewis, Phase Behavior and 3D Structure of Strongly Attractive Microsphere-Nanoparticle Mixtures. Langmuir, 2007. 21(24): p. 1104011047.

30. Barr, S.A. and E. Luijten, Effective Interactions in Mixtures of Silica Microspheres and Polystyrene Nanoparticles. Langmuir, 2006. 22(17): p. 7152-7155.

31. Chang, Y.-I., C.-C. Chang, and W.-Y. Cheng, Can nanoparticles stabilize microparticle suspension? Sep Purif Technol, 2011. 79: p. 393-398.

32. Hoggard, J.D., P.J. Sides, and D.C. Prieve, Measurement of the Streaming Potential and Streaming Current near a Rotating Disk to Determine Its Zeta Potential. Langmuir, 2005. 21(16): p. 7433-7438.

33. Scales, P.J., F. Grieser, and T.W. Healy, Electrokinetics of the Silica-Solution Interface: A Flat Plate Streaming Potential Study. Langmuir, 1992. 8(3): p. 965-974.

34. Henry, D.C., The Cataphoresis of Suspended Particles. Part I. The Equation of Cataphoresis. P Roy Soc A-Math Phy, 1931. 133(821): p. 106-129.

35. Wiersema, P.H., A.L. Loeb, and J.T.G. Overbeek, Calculation of the Electrophoretic Mobility of a Spherical Colloid Particle. J Colloid Interf Sci, 1966. 22: p. 78-99.

36. Heller, W., H.L. Bhatnagar, and M. Nakagaki, Theoretical investigations ont he light scattering of spheres. XIII. The "wavelength exponent" of differential turbidity spectra. J Chem Phys, 1962. 36(5): p. 1163-1170.

37. Long, J.A., D.W.J. Osmond, and B. Vincent, The Equilibrium Aspects of Weak Flocculation. J Colloid Interf Sci, 1973. 42(3): p. 545-553.

38. Sauerbrey, G., Use of quartz vibrator for weighting thin layers and as a microbalance. Z. Phys, 1959. 155: p. 206-222.

39. Anderson, J.L., Effect of Nonuniform Zeta Potential on Particle Movement in Electric Fields. J. Colloid Interface Sci., 1985. 105(1): p. 45-54.

40. Jones, J., G. Holtzer, C. Snyder, A. Yake, and D. Velegol, Charge nonuniformity light scattering. Colloid Surface A, 2005. 267(1-3): p. 79-85.

41. Duffadar, R., S. Kalasin, J.M. Davis, and M.M. Santore, The impact of nanoscale chemical features on micron-scale adhesion: crossover from heterogeneity-dominated to mean-field behavior. J Colloid Interf Sci, 2009. 337(2): p. 396-407.

42. Santore, M.M. and N. Kozlova, Micrometer Scale Adhesion on Nanometer-Scale Patchy Surfaces: Adhesion Rates, Adhesion Thresholds, and Curvature-Based Selectivity. Langmuir, 2007. 23(9): p. 4782-4791. 
43. Tobiason, J.E., Chemical Effects on the Deposition of Non-Brownian Particles. Colloid Surface 1989. 39: p. 53-77.

44. Elimelech, M. and C.R. O'Melia, Effect of Particle Size on Collision Efficiency in the Deposition of Brownian Particles with Electrostatic Energy Barriers. Langmuir, 1990. 6(6): p. 1153-1163. 


\section{Appendix}

\section{Appendix A: Zeta Spin Data}

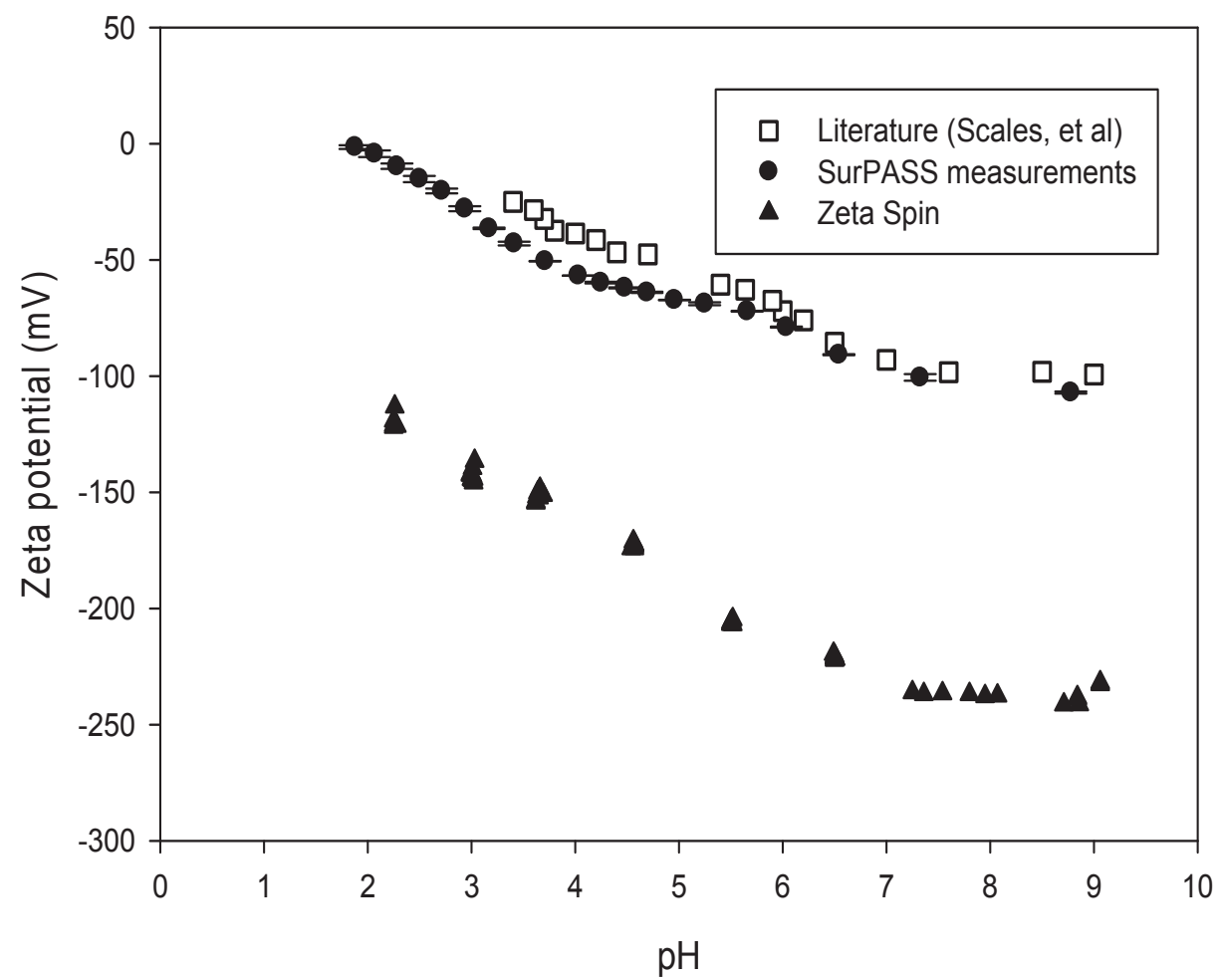

Figure A1: This figure shows a comparison of the zeta potential measurements of flat silica slides. The ZetaSpin values were consistently offset by over $100 \mathrm{mV}$ compared to the SurPASS and literature values, which reasonably agreed [33]. 


\section{Appendix B: TEM images of sulfate and amidine latex particles}

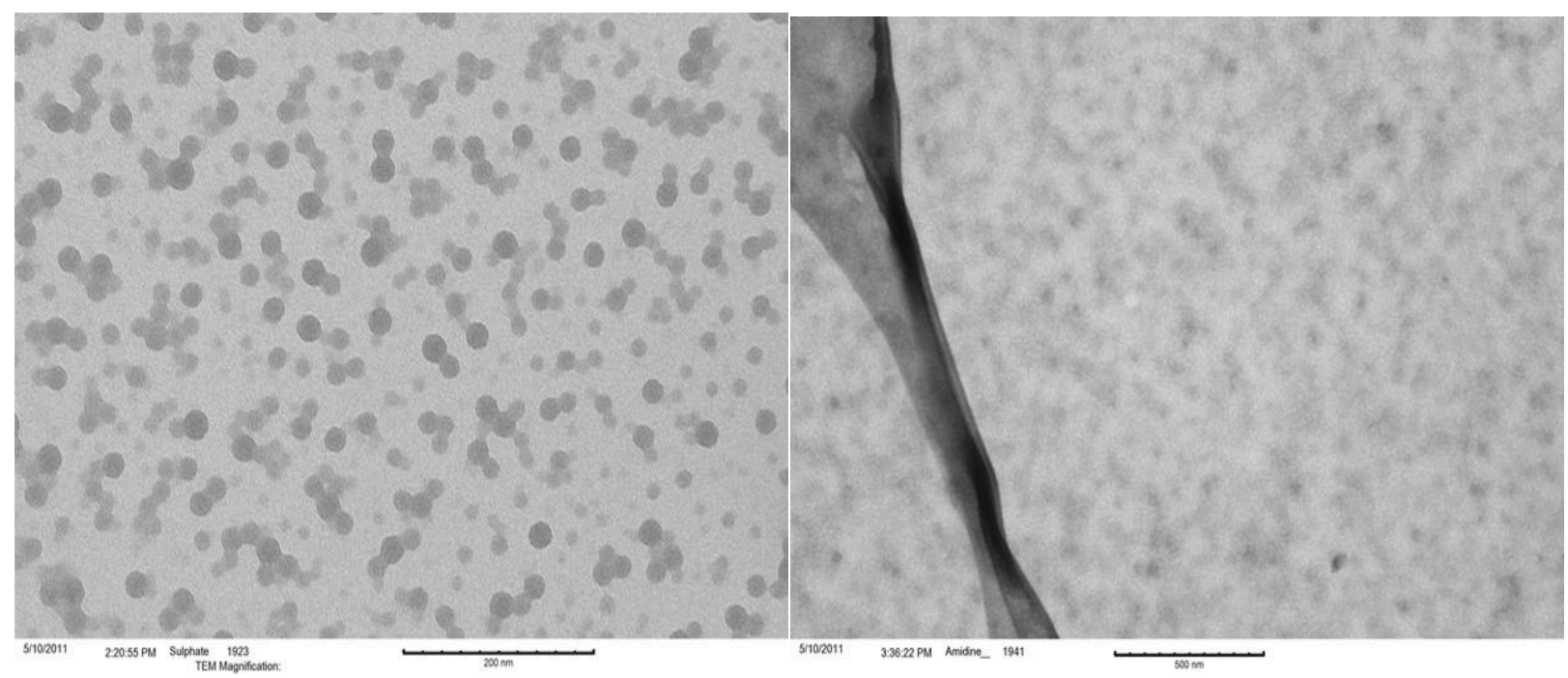

Figure B1: This figure shows TEM images of sulfate latex nanoparticles (left) and amidine latex nanoparticles (right). The Zetasizer had difficulty in determining the size distribution of the amidine particles, so TEM images of the particles were taken to determine if there were significant differences between the size distributions of the two particle types. However, it was impossible to get a clear, properly contrasted image of the amidine particles. This suggests that there is some fundamental difference in the chemistry of the particles that leads to the differences in adsorption behavior. 